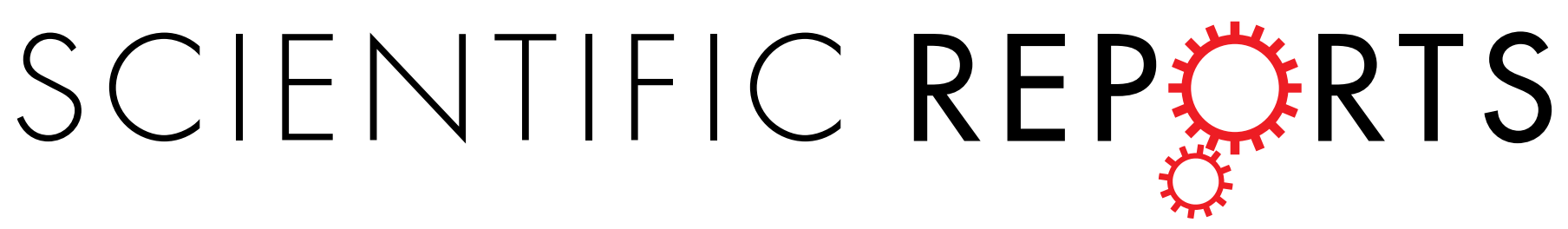

Received: 2 March 2018

Accepted: 13 March 2019

Published online: 09 May 2019

\section{Association between IL-37 gene polymorphisms and risk of HBV- related liver disease in a Saudi Arabian population}

Mashael R. Al-Anazi ${ }^{1}$, Sabine Matou-Nasri ${ }^{2}$, Arwa A. Al-Oahtani ${ }^{3}$, Jahad Alghamdi ${ }^{2}$, Ayman A. Abdo ${ }^{4,9}$, Faisal M. Sanai ${ }^{5,9}$, Waleed K. Al-Hamoudi ${ }^{4,9}$, Khalid A. Alswat ${ }^{4,9}$, Hamad I. Al-Ashgar ${ }^{6}$, Mohammed Q. Khan ${ }^{6}$, Ali Albenmousa ${ }^{7}$, Monis B. Shamsi ${ }^{8}$, Salah K. Alanazi ${ }^{1}$, Damian Dela Cruz ${ }^{1}$, Marie Fe F. Bohol ${ }^{1}$, Mohammed N. Al-Ahdal ${ }^{1,10}$ \& Ahmed A. Al-Qahtani ${ }^{1,10}$

Interleukin-37 (IL-37) has recently been recognized as a strong anti-inflammatory cytokine having anti-tumor activity against hepatocellular carcinoma (HCC) in hepatitis B virus (HBV)-infected patients. $\mathrm{HCC}$ is a typical inflammation-related cancer, and genetic variations within the $I L-37$ gene may be associated with the risk of HBV infection. Identification of the allelic patterns that genetically have a high disease risk is essential for the development of preventive diagnostics for HBV-mediated liver disease pathogenesis. In this study, we aimed to investigate the association between single nucleotide polymorphisms (SNPs) within the IL-37 gene and disease sequelae associated with HBV infection. We genotyped ten IL-37 SNPs in 1274 patients infected with HBV and 599 healthy controls from a Saudi Arabian population. Among the selected SNPs, two SNPs (rs2723175 and rs2708973) were strongly associated with HBV infection, and six SNPs (rs2723176, rs2723175, rs2723186, rs364030, rs28947200, rs4392270) were associated with HBV clearance, comparing healthy controls and HBV infected-patients respectively. A suggestive association of rs 4849133 was identified with active HBV surface antigen ( $\mathrm{HBsAg}$ ) carrier and HBV-related liver disease progression. In conclusion, our findings suggest that variations at the $I L-37$ gene may be useful as genetic predictive risk factors for HBV infection and HBVmediated liver disease progression in the Saudi Arabian population.

Hepatitis B virus (HBV) is a blood-borne virus that specifically infects the liver and triggers immune-mediated liver injury, which may result in cirrhosis or hepatocellular carcinoma (HCC) in extreme cases ${ }^{1}$. HBV is vertically transmitted from infected mothers to their offspring, though it is more frequently acquired through horizontal transmission including contaminated blood transfusion, parenteral routes or via sexual interaction ${ }^{2,3}$. HBV infection affects more than 2 billion people globally, with a high prevalence in Sub-Saharan African and Southeast Asian countries including Saudi Arabia ${ }^{2,4}$. In approximately $90 \%$ of cases, HBV infection is acute, and the virus is cleared within 6 months by the natural immune response ${ }^{5,6}$. Most patients chronically infected with HBV are

${ }^{1}$ Department of Infection and Immunity, Research Center, King Faisal Specialist Hospital \& Research Center, Riyadh, Saudi Arabia. ${ }^{2}$ Medical Genomics Research Department, King Abdullah International Medical Research Center, King Saud bin Abdulaziz University for Health Sciences, Ministry of National Guard Health Affairs, Riyadh, Saudi Arabia. ${ }^{3}$ Department of Family Medicine, Prince Mohammed Bin Abdul Aziz Hospital, Riyadh, Saudi Arabia. ${ }^{4}$ Section of Gastroenterology, Department of Medicine, College of Medicine, King Saud University, Riyadh, Saudi Arabia. ${ }^{5}$ Gastroenterology Unit, Department of Medicine, King Abdulaziz Medical City, Jeddah, Saudi Arabia. ${ }^{6}$ Gastroenterology Unit, Department of Medicine, King Faisal Specialist Hospital \& Research Center, Riyadh, Saudi Arabia. ${ }^{7}$ Department of Gastroenterology, Prince Sultan Medical Military City, Riyadh, Saudi Arabia. ${ }^{8}$ Centre for Genetics and Inherited Diseases, College of Medicine, Taibah University, Madinah, Saudi Arabia. ${ }^{9}$ Liver Disease Research Center, King Saud University, Riyadh, Saudi Arabia. ${ }^{10}$ Department of Microbiology and Immunology, Alfaisal University School of Medicine, Riyadh, Saudi Arabia. Correspondence and requests for materials should be addressed to A.A.A.-Q. (email: aqahtani@kfshrc.edu.sa) 


\begin{tabular}{|c|c|c|c|c|c|c|c|}
\hline Variable & Inactive $(n=563)$ & Active $(n=217)$ & Cirrhosis $(n=64)$ & HCC $(n=30)$ & Healthy Control $(n=599)$ & Clearance $(n=400)$ & p-value ${ }^{a}$ \\
\hline Age (yrs.)** & $40.65 \pm 13.33$ & $36.085 \pm 11.76$ & $53.17 \pm 12.67$ & $60.034 \pm 11.78$ & $30.79 \pm 8.93$ & $37.14 \pm 10.72$ & $<0.0001$ \\
\hline Male count (\%) & $380(67.5 \%)$ & $174(80.2 \%)$ & $51(79.7 \%)$ & $29(96.7 \%)$ & $567(94.7 \%)$ & & $<0.0001$ \\
\hline BMI* & $27.70(24.87-31.73)$ & $27.10(23.06-30.855)$ & $26.14(21.815-29.87)$ & $24.055(21.960-27.44)$ & & & 0.001 \\
\hline $\mathrm{ALT}^{* *}$ & $58.74 \pm 293.82$ & $91.13 \pm 108.10$ & $71.32 \pm 116.04$ & $80.72 \pm 76.44$ & & & 0.157 \\
\hline
\end{tabular}

Table 1. Demographic and clinical characteristics of patients infected with HBV and healthy control subjects. *Values are expressed as median interquartile range (25th-75th), **Values are expressed as Mean \pm SD. $\mathrm{p}^{\mathrm{a}}$ : nonparametric test and one-way ANOVA for continuous data and Chi square test for categorical data. Abbreviations: BMI: Body mass index; ALT: alanine aminotransferase.

asymptomatic and characterized by the absence of the $\mathrm{HB}$ e antigen (HBeAg). The presence of anti-HBe antibodies is associated with largely intact liver tissue ${ }^{2}$. Based on available age-related epidemiological data, in $90 \%$ of infants and $50 \%$ of young children affected with $\mathrm{HBV}$, the infection becomes chronic and persists for many years $^{7,8}$.

Belonging to the Hepadnaviridae family that replicates in human hepatocytes, HBV is an enveloped non-cytopathic virus containing a partially double-stranded viral DNA genome of 3.2-kb length within its core ${ }^{9,10}$. After infecting hepatocytes, HBV releases its genome into the cell host nucleus for viral RNA transcription, DNA replication, and viral protein synthesis including HBV surface antigen (HBsAg). The degree of severity of HBV infection is influenced by several factors such as the age at infection, longer duration of infection, immune status, HBV genotype, high degree of viral mutations, high level of HBV replication, co-infection with hepatitis $\mathrm{C}$ or delta virus, or with human immunodeficiency virus (HIV), male gender, environmental factors (e.g., alcohol consumption, smoking and exposure to aflatoxin), and ethnic background ${ }^{11-16}$.

Recent studies provided additional evidence of the pivotal role of inflammation in patients with chronic HBV infection, which may result in cirrhosis following secondary necroinflammation with the eventual progression to $\mathrm{HCC}^{17,18}$. Pro-inflammatory mediators, such as interferons and cytokines, are produced after the binding of the HBV core protein to membrane heparin sulfate exposed on the cell surface of human hepatoma cells ${ }^{19,20}$. Interleukin-37 (IL-37), a member of the IL-1 family, is an anti-inflammatory cytokine produced by immune cells and suppresses the production of inflammatory cytokines in several types of disease. It has been shown that IL-37 is capable of reducing the activity of both innate and specific immune responses ${ }^{21,22}$. Zhao et al. (2014) showed that decreased expression of IL-37 was correlated with HCC progression ${ }^{23}$, and elevated serum IL-37 levels have been observed in patients infected with HBV and treated with telbivudine ${ }^{24}$. Several studies have shown that there is a significant association between certain genetic variations within the $I L-37$ gene and several diseases, including tuberculosis ${ }^{25,26}$, coronary artery disease (CAD) ${ }^{27}$, and autoimmune-based thyroid diseases ${ }^{28}$.

Despite the implementation of anti-HBV immunization programs for newborns, there are still approximately 5,000 new patients diagnosed with HBV infection per year in Saudi Arabia ${ }^{4,29}$. In this study, we investigated the association between $I L-37$ SNPs and disease sequelae associated with HBV infection in a Saudi Arabian population.

\section{Materials and Methods}

Patients. Peripheral blood samples were collected from 1274 patients infected with HBV and 599 normal healthy volunteers of Saudi origin from three major hospitals in Riyadh City, including King Faisal Specialist Hospital and Research Center (KFSHRC), King Khalid University Hospital (KKUH), and Prince Sultan Military Medical City (PSMMC). Written informed consent was obtained from participating individuals, and the study was approved by the institutional review board of the participating hospitals in accordance with the Helsinki Declaration of 1975. The patients were grouped in five categories based on disease severity: group I included patients who cleared HBV $(n=400)$, group II patients with inactive HBV infection $(n=563)$, group III patients with active HBV infection $(n=217)$, group IV patients with $\mathrm{HBV}$-associated cirrhosis $(\mathrm{n}=64)$, and group V patients with cirrhosis diagnosed with HCC $(n=30)$. Control subjects were characterized by the absence of any known serological marker for HBV.

TagSNP Selection. The SNP data of the entire $I L-37$ gene were downloaded from the 1000 Genomes Project Database (GPD; http://www.internationalgenome.org). All genetic variants with a minor allele frequency $\geq 0.05$ and located within the IL37 genomic region (Chromosome 2: 113,670,548-113,676,459, GRCh37) plus a flanking region of $7 \mathrm{~kb}$ were extracted from the 1000 Genome Project - Phase $3^{30,31}$. The Tagger tool as implemented in Haploview Software (Broad Institute of MIT and Harvard, Cambridge, MA, USA, version 4.2) was used to select tag SNPs that span this genomic region using the pairwise tagging method and an $r^{2}$ threshold of 0.8. Of the identified 134 variants in the $1000 \mathrm{GPD}$, we selected 10 tag SNPs that captures $103(76 \%)$ alleles at $r^{2} \geq 0.8$ with a mean $\max r^{2}$ equal to 0.925 . The final set of SNPs investigated in this study was rs2723176, rs2723175, rs2723186, rs2723168, rs4364030, rs3811047, rs28947200, rs4392270, rs4849133, and rs2708973. 


\begin{tabular}{|c|c|c|c|c|c|c|c|c|}
\hline SNPs & $\begin{array}{l}\text { Genotype/Allele } \\
\text { distribution }\end{array}$ & \begin{tabular}{|l|}
$\begin{array}{l}\text { Control } \\
(\mathrm{n}=599)\end{array}$ \\
\end{tabular} & $\%$ & $\begin{array}{l}\text { HBV patients } \\
(\mathrm{n}=874)\end{array}$ & $\%$ & OR (95\% C.I.) & $x^{2}$ & p-value \\
\hline \multirow{7}{*}{ rs2723176 } & CC & 560 & $93.49 \%$ & 794 & $90.85 \%$ & Ref & & \\
\hline & AC & 36 & $6.01 \%$ & 75 & $8.58 \%$ & $1.469(0.973-2.218)$ & 3.387 & 0.066 \\
\hline & AA & 3 & $0.50 \%$ & 5 & $0.57 \%$ & $1.175(0.280-4.939$ & 0.049 & 1.00 \\
\hline & $\mathrm{C}$ & 1156 & $96.49 \%$ & 1663 & $95.14 \%$ & \multirow{2}{*}{$1.407(0.965-2.051)$} & \multirow{2}{*}{3.172} & \multirow{2}{*}{0.075} \\
\hline & A & 42 & $3.51 \%$ & 85 & $4.86 \%$ & & & \\
\hline & $\mathrm{AA}+\mathrm{AC}$ vs $\mathrm{CC}$ & & & & & $1.447(0.972-2.153)$ & 3.340 & 0.068 \\
\hline & $\mathrm{AA}$ vs $\mathrm{AC}+\mathrm{CC}$ & & & & & $0.875(0.208-3.675)$ & 0.033 & 0.854 \\
\hline \multirow{7}{*}{ rs 2723175} & GG & 554 & $92.49 \%$ & 591 & $67.62 \%$ & Ref & & \\
\hline & AG & 28 & $4.67 \%$ & 238 & $27.23 \%$ & 7.968 (5.296-11.987) & 127.66 & $<0.0001$ \\
\hline & AA & 17 & $2.84 \%$ & 45 & $5.15 \%$ & $2.481(1.403-4.387)$ & 10.370 & 0.001 \\
\hline & G & 1136 & $94.82 \%$ & 1420 & $81.24 \%$ & \multirow{2}{*}{$4.232(3.191-5.613)$} & \multirow{2}{*}{114.283} & \multirow{2}{*}{$<0.0001$} \\
\hline & A & 62 & $5.18 \%$ & 328 & $18.76 \%$ & & & \\
\hline & $\mathrm{AA}+\mathrm{AG}$ vs GG & & & & & $5.895(4.216-8.243)$ & 126.976 & $<0.0001$ \\
\hline & $\mathrm{AA}$ vs $\mathrm{AG}+\mathrm{GG}$ & & & & & $0.538(0.305-0.950)$ & 4.710 & 0.030 \\
\hline \multirow{7}{*}{ rs2723186 } & GG & 553 & $92.32 \%$ & 782 & $89.47 \%$ & Ref & & \\
\hline & AG & 28 & $4.67 \%$ & 54 & $6.18 \%$ & $1.364(0.853-2.180)$ & 1.691 & 0.193 \\
\hline & AA & 18 & $3.01 \%$ & 38 & $4.35 \%$ & $1.493(0.843-2.643)$ & 1.913 & 0.167 \\
\hline & G & 1134 & $94.66 \%$ & 1618 & $92.56 \%$ & \multirow{2}{*}{$1.424(1.045-1.939)$} & \multirow{2}{*}{5.071} & \multirow{2}{*}{0.024} \\
\hline & $\mathrm{A}$ & 64 & $5.34 \%$ & 130 & $7.44 \%$ & & & \\
\hline & $\mathrm{AA}+\mathrm{AG}$ vs GG & & & & & $1.414(0.977-2.048)$ & 3.392 & 0.065 \\
\hline & $\mathrm{AA}$ vs $\mathrm{AG}+\mathrm{GG}$ & & & & & $0.682(0.385-1.206)$ & 1.752 & 0.186 \\
\hline \multirow{7}{*}{ rs2723168 } & AA & 19 & $3.17 \%$ & 35 & $4.00 \%$ & Ref & & \\
\hline & AG & 578 & $96.49 \%$ & 834 & $95.42 \%$ & $0.783(0.444-1.383)$ & 0.712 & 0.399 \\
\hline & GG & 2 & $0.33 \%$ & 5 & $0.57 \%$ & $1.357(0.240-7.673)$ & 0.120 & 0.728 \\
\hline & $\mathrm{A}$ & 616 & $51.42 \%$ & 904 & $51.72 \%$ & \multirow{2}{*}{$0.988(0.853-1.145)$} & \multirow{2}{*}{0.025} & \multirow{2}{*}{0.874} \\
\hline & G & 582 & $48.58 \%$ & 844 & $48.28 \%$ & & & \\
\hline & GG + AG vs AA & & & & & $0.785(0.445-1.386)$ & 0.698 & 0.404 \\
\hline & GG vs AG + AA & & & & & $0.582(0.113-3.011)$ & 0.426 & 0.708 \\
\hline & CC & 292 & $48.75 \%$ & 428 & $48.97 \%$ & Ref & & \\
\hline & CG & 245 & $40.90 \%$ & 357 & $40.85 \%$ & $0.994(0.797-1.239)$ & 0.003 & 0.958 \\
\hline & GG & 62 & $10.35 \%$ & 89 & $10.18 \%$ & $0.979(0.686-1.399)$ & 0.013 & 0.909 \\
\hline rs4364030 & C & 829 & $69.20 \%$ & 1213 & $69.39 \%$ & $0991(0845-1162)$ & 0013 & 0910 \\
\hline & G & 369 & $30.80 \%$ & 535 & $30.61 \%$ & $0.991(0.845-1.162)$ & 0.013 & 0.910 \\
\hline & GG + CG vs CC & & & & & $0.991(0.805-1.220)$ & 0.007 & 0.933 \\
\hline & GG vs CG + CC & & & & & $1.018(0.723-1.434)$ & 0.011 & 0.917 \\
\hline & GG & 168 & $28.05 \%$ & 264 & $30.21 \%$ & Ref & & \\
\hline & AG & 278 & $46.41 \%$ & 389 & $44.51 \%$ & $0.890(0.695-1.140)$ & 0.850 & 0.358 \\
\hline & AA & 153 & $25.54 \%$ & 221 & $25.29 \%$ & $0.919(0.693-1.219)$ & 0.340 & 0.559 \\
\hline rs3811047 & G & 614 & $51.25 \%$ & 917 & $52.46 \%$ & $0953(0822101$ & 0400 & 0510 \\
\hline & A & 584 & $48.75 \%$ & 831 & $47.54 \%$ & $0.953(0.822-1.104)$ & 0.420 & 0.519 \\
\hline & $\mathrm{AA}+\mathrm{AG}$ vs GG & & & & & $0.901(0.716-1.133)$ & 0.800 & 0.371 \\
\hline & AA vs AG + GG & & & & & $1.014(0.798-1.287)$ & 0.010 & 0.912 \\
\hline & CC & 511 & $85.31 \%$ & 771 & $88.22 \%$ & Ref & & \\
\hline & CT & 55 & $9.18 \%$ & 80 & $9.15 \%$ & $0.964(0.672-1.383)$ & 0.040 & 0.842 \\
\hline & TT & 33 & $5.51 \%$ & 23 & $2.63 \%$ & $0.462(0.268-0.796)$ & 8.087 & 0.004 \\
\hline rs 28947200 & $\mathrm{C}$ & 1077 & $89.90 \%$ & 1622 & $92.79 \%$ & & & \\
\hline & $\mathrm{T}$ & 121 & $10.10 \%$ & 126 & $7.21 \%$ & $0.691(0.533-0.898)$ & 7.739 & 0.005 \\
\hline & TT + CT vs CC & & & & & $0.776(0.571-1.053)$ & 2.660 & 0.103 \\
\hline & TT vs CT + CC & & & & & $2.157(1.254-3.713)$ & 8.047 & 0.005 \\
\hline & GG & 555 & $92.65 \%$ & 796 & $91.08 \%$ & Ref & & \\
\hline & AG & 33 & $5.51 \%$ & 54 & $6.18 \%$ & $1.141(0.730-1.783)$ & 0.335 & 0.562 \\
\hline & $\mathrm{AA}$ & 11 & $1.84 \%$ & 24 & $2.75 \%$ & $1.521(0.739-3.131)$ & 1.316 & 0.251 \\
\hline rs4392270 & G & 1143 & $95.41 \%$ & 1646 & $94.16 \%$ & & & \\
\hline & A & 55 & $4.59 \%$ & 102 & $5.84 \%$ & $1.288(0.920-1.803)$ & 2.181 & 0.139 \\
\hline & $\mathrm{AA}+\mathrm{AG}$ vs $\mathrm{GG}$ & & & & & $1.236(0.8841-1.817)$ & 1.166 & 0.280 \\
\hline & $\mathrm{AA}$ vs $\mathrm{AG}+\mathrm{GG}$ & & & & & $0.663(0.322-1.363)$ & 1.268 & 0.260 \\
\hline
\end{tabular}




\begin{tabular}{|c|c|c|c|c|c|c|c|c|}
\hline SNPs & $\begin{array}{l}\text { Genotype/Allele } \\
\text { distribution }\end{array}$ & \begin{tabular}{|l} 
Control \\
$(\mathrm{n}=599)$
\end{tabular} & $\%$ & $\begin{array}{l}\text { HBV patients } \\
(\mathbf{n}=874)\end{array}$ & $\%$ & OR (95\% C.I.) & $x^{2}$ & p-value \\
\hline \multirow{7}{*}{ rs4849133 } & TT & 513 & $85.64 \%$ & 706 & $80.78 \%$ & Ref & & \\
\hline & CT & 67 & $11.19 \%$ & 119 & $13.62 \%$ & $1.291(0.937-1.778)$ & 2.447 & 0.118 \\
\hline & $\mathrm{CC}$ & 19 & $3.17 \%$ & 49 & $5.61 \%$ & $1.874(1.090-3.221)$ & 5.312 & 0.021 \\
\hline & $\mathrm{T}$ & 1093 & $91.24 \%$ & 1531 & $87.59 \%$ & \multirow{2}{*}{$1.475(1.154-1.886)$} & \multirow{2}{*}{9.725} & \multirow{2}{*}{0.002} \\
\hline & $\mathrm{C}$ & 105 & $8.76 \%$ & 217 & $12.41 \%$ & & & \\
\hline & $\mathrm{CC}+\mathrm{CT}$ vs TT & & & & & $1.419(1.069-1.885)$ & 5.894 & 0.015 \\
\hline & $\mathrm{CC}$ vs CT $+\mathrm{TT}$ & & & & & $0.552(0.321-0.947)$ & 4.784 & 0.029 \\
\hline \multirow{7}{*}{ rs2708973 } & GG & 576 & $96.16 \%$ & 787 & $90.05 \%$ & Ref & & \\
\hline & AG & 9 & $1.50 \%$ & 65 & $7.44 \%$ & $5.286(2.611-10.701)$ & 26.342 & $<0.0001$ \\
\hline & AA & 14 & $2.34 \%$ & 22 & $2.52 \%$ & $1.150(0.583-2.267)$ & 0.163 & 0.686 \\
\hline & G & 1161 & $96.91 \%$ & 1639 & $93.76 \%$ & \multirow{2}{*}{$2.087(1.426-3.053)$} & \multirow{2}{*}{14.948} & \multirow{2}{*}{$<0.0001$} \\
\hline & A & 37 & $3.09 \%$ & 109 & $6.24 \%$ & & & \\
\hline & $\mathrm{AA}+\mathrm{AG}$ vs GG & & & & & $2.768(1.727-4.438)$ & 19.230 & $<0.0001$ \\
\hline & $\mathrm{AA}$ vs $\mathrm{AG}+\mathrm{GG}$ & & & & & $0.927(0.470-1.826)$ & 0.050 & 0.826 \\
\hline
\end{tabular}

Table 2. Comparison of genotypic distributions between patients infected with HBV and healthy controls. Bold indicates significance.

\begin{tabular}{|c|c|c|c|c|c|c|c|c|}
\hline SNPs & $\begin{array}{l}\text { Genotype/Allele } \\
\text { distribution }\end{array}$ & $\begin{array}{l}\text { Clearance } \\
(\mathrm{n}=400)\end{array}$ & $\%$ & $\begin{array}{l}\text { HBV patients } \\
(\mathrm{n}=874)\end{array}$ & $\%$ & OR (95\% C.I.) & $x^{2}$ & p-value \\
\hline \multirow{7}{*}{ rs2723176 } & $\mathrm{CC}$ & 335 & $83.75 \%$ & 794 & $90.85 \%$ & Ref & & \\
\hline & $\mathrm{AC}$ & 58 & $14.50 \%$ & 75 & $8.58 \%$ & $0.546(0.378-0.786)$ & 10.780 & 0.001 \\
\hline & AA & 7 & $1.75 \%$ & 5 & $0.57 \%$ & $0.301(0.095-0.956)$ & 4.650 & 0.031 \\
\hline & $\mathrm{C}$ & 728 & $91.00 \%$ & 1663 & $95.14 \%$ & \multirow{2}{*}{$0.517(0.373-0.716)$} & \multirow{2}{*}{16.250} & \multirow{2}{*}{$<0.0001$} \\
\hline & $\mathbf{A}$ & 72 & $9.00 \%$ & 85 & $4.86 \%$ & & & \\
\hline & $\mathbf{A A}+\mathbf{A C}$ vs $\mathbf{C C}$ & & & & & $0.519(0.365-0.738)$ & 13.700 & 0.0002 \\
\hline & $\mathrm{AA}$ vs $\mathrm{AC}+\mathrm{CC}$ & & & & & $3.096(0.976-9.814)$ & 4.080 & 0.043 \\
\hline \multirow{7}{*}{ rs2723175 } & GG & 369 & $92.25 \%$ & 591 & $67.62 \%$ & Ref & & \\
\hline & AG & 14 & $3.50 \%$ & 238 & $27.23 \%$ & $10.614(6.097-18.480)$ & 99.850 & $<0.0001$ \\
\hline & $\mathrm{AA}$ & 17 & $4.25 \%$ & 45 & $5.15 \%$ & $1.653(0.932-2.931)$ & 3.010 & 0.083 \\
\hline & G & 752 & $94.00 \%$ & 1420 & $81.24 \%$ & \multirow{2}{*}{$3.619(2.640-4.961)$} & \multirow{2}{*}{71.080} & \multirow{2}{*}{$<0.0001$} \\
\hline & $\mathbf{A}$ & 48 & $6.00 \%$ & 328 & $18.76 \%$ & & & \\
\hline & AA + AG vs GG & & & & & $5.700(3.848-8.443)$ & 89.630 & $<0.0001$ \\
\hline & AA vs $\mathrm{AG}+\mathrm{GG}$ & & & & & $0.818(0.462-1.447)$ & 0.480 & 0.489 \\
\hline \multirow{7}{*}{ rs2723186 } & GG & 331 & $82.75 \%$ & 782 & $89.47 \%$ & Ref & & \\
\hline & AG & 62 & $15.50 \%$ & 54 & $6.18 \%$ & $0.369(0.250-0.543)$ & 27.150 & $<0.0001$ \\
\hline & AA & 7 & $1.75 \%$ & 38 & $4.35 \%$ & $2.298(1.016-5.198)$ & 4.210 & 0.040 \\
\hline & G & 724 & $90.50 \%$ & 1618 & $92.56 \%$ & \multirow{2}{*}{$0.765(0.569-1.029)$} & \multirow{2}{*}{3.140} & \multirow{2}{*}{0.076} \\
\hline & A & 76 & $9.50 \%$ & 130 & $7.44 \%$ & & & \\
\hline & AA + AG vs GG & & & & & $0.564(0.403-0.791)$ & 11.240 & 0.0008 \\
\hline & AA vs $A G+G G$ & & & & & $0.392(0.173-0.885)$ & 5.430 & 0.019 \\
\hline \multirow{7}{*}{ rs 2723168} & AA & 4 & $1.00 \%$ & 35 & $4.00 \%$ & Ref & & \\
\hline & AG & 393 & $98.25 \%$ & 834 & $95.42 \%$ & $0.243(0.086-0.687)$ & 8.320 & 0.004 \\
\hline & GG & 3 & $0.75 \%$ & 5 & $0.57 \%$ & $0.190(0.033-1.114)$ & 3.890 & 0.049 \\
\hline & $\mathrm{A}$ & 401 & $50.13 \%$ & 904 & $51.72 \%$ & \multirow{2}{*}{$0.938(0.794-1.109)$} & \multirow{2}{*}{0.560} & \multirow{2}{*}{0.456} \\
\hline & G & 399 & $49.88 \%$ & 844 & $48.28 \%$ & & & \\
\hline & GG + AG vs AA & & & & & $0.242(0.085-0.686)$ & 8.350 & 0.004 \\
\hline & GG vs AG + AA & & & & & $1.313(0.312-5.523)$ & 0.140 & 0.709 \\
\hline \multirow{7}{*}{ rs4364030 } & $\mathrm{CC}$ & 162 & $40.50 \%$ & 428 & $48.97 \%$ & Ref & & \\
\hline & CG & 171 & $42.75 \%$ & 357 & $40.85 \%$ & $0.790(0.611-1.022)$ & 3.240 & 0.072 \\
\hline & GG & 67 & $16.75 \%$ & 89 & $10.18 \%$ & $0.503(0.349-0.724)$ & 13.920 & 0.0002 \\
\hline & C & 495 & $61.88 \%$ & 1213 & $69.39 \%$ & \multirow{2}{*}{$0.716(0.601-0.853)$} & \multirow{2}{*}{14.040} & \multirow{2}{*}{0.0002} \\
\hline & G & 305 & $38.13 \%$ & 535 & $30.61 \%$ & & & \\
\hline & GG + CG vs CC & & & & & $0.709(0.558-0.901)$ & 7.920 & 0.005 \\
\hline & GG vs CG + CC & & & & & $1.313(0.312-5.523)$ & 0.140 & 0.709 \\
\hline \multicolumn{9}{|c|}{ Continued } \\
\hline
\end{tabular}




\begin{tabular}{|c|c|c|c|c|c|c|c|c|}
\hline SNPs & $\begin{array}{l}\text { Genotype/Allele } \\
\text { distribution }\end{array}$ & $\begin{array}{l}\text { Clearance } \\
(\mathrm{n}=400)\end{array}$ & $\%$ & $\begin{array}{l}\text { HBV patients } \\
(\mathrm{n}=874)\end{array}$ & $\%$ & OR (95\% C.I.) & $x^{2}$ & p-value \\
\hline \multirow{7}{*}{ rs3811047 } & GG & 139 & $34.75 \%$ & 264 & $30.21 \%$ & Ref & & \\
\hline & AG & 176 & $44.00 \%$ & 389 & $44.51 \%$ & $1.164(0.887-1.527)$ & 1.200 & 0.274 \\
\hline & AA & 85 & $21.25 \%$ & 221 & $25.29 \%$ & $1.369(0.990-1.892)$ & 3.630 & 0.057 \\
\hline & G & 454 & $56.75 \%$ & 917 & $52.46 \%$ & \multirow{2}{*}{$1.189(1.005-1.407)$} & \multirow{2}{*}{4.060} & \multirow{2}{*}{0.044} \\
\hline & A & 346 & $43.25 \%$ & 831 & $47.54 \%$ & & & \\
\hline & $\mathrm{AA}+\mathrm{AG}$ vs GG & & & & & $1.231(0.957-1.582)$ & 2.620 & 0.106 \\
\hline & AA vs $A G+G G$ & & & & & $0.797(0.600-1.059)$ & 2.450 & 0.118 \\
\hline \multirow{7}{*}{ rs2894720 } & CC & 393 & $98.25 \%$ & 771 & $88.22 \%$ & Ref & & \\
\hline & CT & 2 & $0.50 \%$ & 80 & $9.15 \%$ & $20.389(4.986-83.377)$ & 34.710 & $<0.0001$ \\
\hline & TT & 5 & $1.25 \%$ & 23 & $2.63 \%$ & $2.345(0.885-6.215)$ & 3.110 & 0.078 \\
\hline & C & 788 & $98.50 \%$ & 1622 & $92.79 \%$ & \multirow{2}{*}{$5.101(2.805-9.278)$} & \multirow{2}{*}{34.910} & \multirow{2}{*}{$<0.0001$} \\
\hline & $\mathrm{T}$ & 12 & $1.50 \%$ & 126 & $7.21 \%$ & & & \\
\hline & TT + CT vs CC & & & & & $7.500(3.455-16.282)$ & 35.030 & $<0.0001$ \\
\hline & TT vs $\mathrm{CT}+\mathrm{CC}$ & & & & & $0.468(0.177-1.241)$ & 2.440 & 0.119 \\
\hline \multirow{7}{*}{ rs4392270 } & GG & 340 & $85.00 \%$ & 796 & $91.08 \%$ & Ref & & \\
\hline & AG & 52 & $13.00 \%$ & 54 & $6.18 \%$ & $0.444(0.297-0.663)$ & 16.420 & $<0.0001$ \\
\hline & AA & 8 & $2.00 \%$ & 24 & $2.75 \%$ & $1.281(0.570-2.881)$ & 0.360 & 0.548 \\
\hline & G & 732 & $91.50 \%$ & 1646 & $94.16 \%$ & \multirow{2}{*}{$0.667(0.485-0.918)$} & \multirow{2}{*}{6.260} & \multirow{2}{*}{0.012} \\
\hline & A & 68 & $8.50 \%$ & 102 & $5.84 \%$ & & & \\
\hline & $\mathrm{AA}+\mathrm{AG}$ vs GG & & & & & $0.555(0.388-0.796)$ & 10.490 & 0.001 \\
\hline & AA vs $\mathrm{AG}+\mathrm{GG}$ & & & & & $0.723(0.322-1.623)$ & 0.620 & 0.429 \\
\hline \multirow{7}{*}{ rs4849133 } & TT & 311 & $77.75 \%$ & 706 & $80.78 \%$ & Ref & & \\
\hline & CT & 77 & $19.25 \%$ & 119 & $13.62 \%$ & $0.681(0.496-0.934)$ & 5.720 & 0.017 \\
\hline & CC & 12 & $3.00 \%$ & 49 & $5.61 \%$ & $1.799(0.944-3.429)$ & 3.260 & 0.071 \\
\hline & \begin{tabular}{|l|}
$\mathrm{T}$ \\
\end{tabular} & 699 & $87.38 \%$ & 1531 & $87.59 \%$ & \multirow{2}{*}{$0.981(0.762-1.263)$} & \multirow{2}{*}{0.020} & \multirow{2}{*}{0.881} \\
\hline & C & 101 & $12.63 \%$ & 217 & $12.41 \%$ & & & \\
\hline & CC + CT vs TT & & & & & $0.832(0.622-1.111)$ & 1.560 & 0.211 \\
\hline & CC vs CT + TT & & & & & $0.521(0.274-0.990)$ & 4.090 & 0.043 \\
\hline \multirow{7}{*}{ rs2708973 } & GG & 364 & $91.00 \%$ & 787 & $90.05 \%$ & Ref & & \\
\hline & AG & 18 & $4.50 \%$ & 65 & $7.44 \%$ & $1.670(0.977-2.856)$ & 3.580 & 0.059 \\
\hline & AA & 18 & $4.50 \%$ & 22 & $2.52 \%$ & $0.565(0.300-1.067)$ & 3.170 & 0.075 \\
\hline & G & 746 & $93.25 \%$ & 1639 & $93.76 \%$ & \multirow{2}{*}{$0.919(0.656-1.287)$} & \multirow{2}{*}{0.240} & \multirow{2}{*}{0.622} \\
\hline & A & 54 & $6.75 \%$ & 109 & $6.24 \%$ & & & \\
\hline & $\mathrm{AA}+\mathrm{AG}$ vs GG & & & & & $1.118(0.743-1.681)$ & 0.290 & 0.593 \\
\hline & $\mathrm{AA}$ vs $\mathrm{AG}+\mathrm{GG}$ & & & & & & & \\
\hline
\end{tabular}

Table 3. Comparison of genotypic distributions between patients infected with HBV and the clearance group. Bold indicates significance.

Genotyping of IL-37 SNPs. Genomic DNA was extracted from the buffy coats isolated from patients with HBV using the Gentra Pure Gene kit (Qiagen, Hilden, Germany). Patient and control samples were genotyped for the ten selected SNPs using the 7900 HT Fast Real Time PCR System (Applied Biosystems, Foster City, CA, USA). The reagents used included universal TaqMan master mix, amplifying primers, and probes specific for each SNP and were purchased from Applied Biosystems. For each SNP, one allelic probe was labeled with FAM dye and the other with fluorescent VIC dye. The reaction was performed in a 96-well plate in a total reaction volume of $25 \mu \mathrm{L}$ using $20 \mathrm{ng}$ of genomic DNA. The TaqMan assay was subsequently read and analyzed by an automated software sequence detection system (SDS, version 2.4.1).

Statistical analysis. Statistical analysis was performed using the SPSS version 20.0 (SPSS Inc., Chicago, IL, USA) and HaploView version 4.2. The association between the $I L-37$ tag SNPs and disease status was expressed in odds ratio (OR) and 95\% confidence intervals (CI). A statistically significant level of association was corrected for multiple testing, and only associations less than 0.00125 were considered significant. The SNPs were tested for the Hardy-Weinberg equilibrium (HWE) using Haploview software. A cut-off p-value of 0.05 was set for the HWE, and SNPs were excluded if they did not meet this value. OR values with CI calculated in fixed or random-effects models were used to estimate the strength of the association.

\section{Results}

Characteristics of the study subjects. Table 1 displays the demographic and clinical details of patients infected with HBV and the control subjects. The analysis shows that older age, male gender, body mass index (BMI), and HBV load were significantly associated with the risk of HBV chronic infection developing into severe liver disease such as cirrhosis and HCC. 


\begin{tabular}{|c|c|c|c|c|c|c|c|c|}
\hline SNPs & $\begin{array}{l}\text { Genotype/Allele } \\
\text { distribution }\end{array}$ & $\begin{array}{l}\text { Inactive } \\
(\mathrm{n}=563)\end{array}$ & $\%$ & $\begin{array}{l}\text { Active, cirrhosis } \\
\text { and HCC }(n=311)\end{array}$ & $\%$ & OR (95\% C.I.) & $x^{2}$ & p-value \\
\hline \multirow{7}{*}{ rs2723176 } & CC & 518 & $92.01 \%$ & 276 & $88.75 \%$ & Ref & & \\
\hline & AC & 42 & $7.46 \%$ & 33 & $10.61 \%$ & $1.475(0.914-2.380)$ & 2.550 & 0.110 \\
\hline & AA & 3 & $0.53 \%$ & 2 & $0.64 \%$ & $1.251(0.208-7.533)$ & 0.060 & 0.806 \\
\hline & C & 1078 & $95.74 \%$ & 585 & $94.05 \%$ & \multirow{2}{*}{$1.420(0.914-2.206)$} & \multirow{2}{*}{2.460} & \multirow{2}{*}{0.117} \\
\hline & A & 48 & $4.26 \%$ & 37 & $5.95 \%$ & & & \\
\hline & $\mathrm{AA}+\mathrm{AC}$ vs CC & & & & & $1.460(0.917-2.325)$ & 2.560 & 0.109 \\
\hline & $\mathrm{AA}$ vs $\mathrm{AC}+\mathrm{CC}$ & & & & & $0.828(0.138-4.980)$ & 0.040 & 0.836 \\
\hline \multirow{7}{*}{ rs2723175 } & GG & 374 & $66.43 \%$ & 217 & $69.77 \%$ & Ref & & \\
\hline & AG & 156 & $27.71 \%$ & 82 & $26.37 \%$ & $0.906(0.661-1.242)$ & 0.380 & 0.539 \\
\hline & AA & 33 & $5.86 \%$ & 12 & $3.86 \%$ & $0.627(0.317-1.239)$ & 1.830 & 0.176 \\
\hline & G & 904 & $80.28 \%$ & 516 & $82.96 \%$ & \multirow{2}{*}{$0.837(0.648-1.080)$} & \multirow{2}{*}{1.880} & \multirow{2}{*}{0.170} \\
\hline & A & 222 & $19.72 \%$ & 106 & $17.04 \%$ & & & \\
\hline & $\mathrm{AA}+\mathrm{AG}$ vs GG & & & & & $0.857(0.636-1.155)$ & 1.020 & 0.312 \\
\hline & $\mathrm{AA}$ vs $\mathrm{AG}+\mathrm{GG}$ & & & & & $1.551(0.789-3.049)$ & 1.650 & 0.199 \\
\hline \multirow{7}{*}{ rs2723186 } & GG & 509 & $90.41 \%$ & 273 & $87.78 \%$ & Ref & & \\
\hline & AG & 30 & $5.33 \%$ & 24 & $7.72 \%$ & $1.492(0.855-2.602)$ & 2.00 & 0.157 \\
\hline & AA & 24 & $4.26 \%$ & 14 & $4.50 \%$ & $1.088(0.554-2.137)$ & 0.060 & 0.807 \\
\hline & G & 1048 & $93.07 \%$ & 570 & $91.64 \%$ & \multirow{2}{*}{$1.226(0.851-1.766)$} & \multirow{2}{*}{1.200} & \multirow{2}{*}{0.274} \\
\hline & A & 78 & $6.93 \%$ & 52 & $8.36 \%$ & & & \\
\hline & $\mathrm{AA}+\mathrm{AG}$ vs GG & & & & & $1.312(0.845-2.038)$ & 1.470 & 0.226 \\
\hline & AA vs $\mathrm{AG}+\mathrm{GG}$ & & & & & $0.945(0.481-1.854)$ & 0.030 & 0.868 \\
\hline \multirow{7}{*}{ rs2723168 } & AA & 23 & $4.09 \%$ & 12 & $3.86 \%$ & Ref & & \\
\hline & AG & 537 & $95.38 \%$ & 297 & $95.50 \%$ & $1.060(0.520-2.161)$ & 0.030 & 0.872 \\
\hline & GG & 3 & $0.53 \%$ & 2 & $0.64 \%$ & $1.278(0.187-8.720)$ & 0.060 & 0.802 \\
\hline & A & 583 & $51.78 \%$ & 321 & $51.61 \%$ & \multirow{2}{*}{$1.007(0.828-1.225)$} & \multirow{2}{*}{0.00} & \multirow{2}{*}{0.946} \\
\hline & G & 543 & $48.22 \%$ & 301 & $48.39 \%$ & & & \\
\hline & GG + AG vs AA & & & & & $1.061(0.521-2.163)$ & 0.030 & 0.869 \\
\hline & GG vs AG + AA & & & & & $0.828(0.138-4.980)$ & 0.040 & 0.836 \\
\hline & CC & 268 & $47.60 \%$ & 160 & $51.45 \%$ & Ref & & \\
\hline & CG & 241 & $42.81 \%$ & 116 & $37.30 \%$ & $0.806(0.600-1.084)$ & 2.040 & 0.153 \\
\hline & GG & 54 & $9.59 \%$ & 35 & $11.25 \%$ & $1.086(0.680-1.734)$ & 0.120 & 0.731 \\
\hline rs 4364030 & C & 777 & $69.01 \%$ & 436 & $70.10 \%$ & $0950(0767-175)$ & 0220 & 0.636 \\
\hline & G & 349 & $30.99 \%$ & 186 & $29.90 \%$ & $0.950(0.767-1.175)$ & 0.220 & 0.636 \\
\hline & GG + CG vs CC & & & & & $0.857(0.650-1.131)$ & 1.190 & 0.276 \\
\hline & GG vs CG + CC & & & & & $0.837(0.534-1.312)$ & 0.610 & 0.436 \\
\hline & GG & 160 & $28.42 \%$ & 123 & $39.55 \%$ & Ref & & \\
\hline & AG & 267 & $47.42 \%$ & 151 & $48.55 \%$ & $0.736(0.540-1.001)$ & 3.820 & 0.051 \\
\hline & AA & 136 & $24.16 \%$ & 101 & $32.48 \%$ & $0.966(0.682-1.369)$ & 0.040 & 0.846 \\
\hline rs3811047 & G & 587 & $52.13 \%$ & \begin{tabular}{|l|}
397 \\
\end{tabular} & $63.83 \%$ & $0968(0805-1165)$ & 0120 & 0733 \\
\hline & A & 539 & $47.87 \%$ & 353 & $56.75 \%$ & $0.968(0.805-1.165)$ & 0.120 & 0.733 \\
\hline & $\mathrm{AA}+\mathrm{AG}$ vs GG & & & & & $0.813(0.613-1.079)$ & 2.050 & 0.152 \\
\hline & AA vs $A G+G G$ & & & & & $0.864(0.641-1.165)$ & 0.920 & 0.338 \\
\hline & $\mathrm{CC}$ & 499 & $88.63 \%$ & 272 & $87.46 \%$ & Ref & & \\
\hline & CT & 49 & $8.70 \%$ & 31 & $9.97 \%$ & $1.161(0.723-1.863)$ & 0.380 & 0.537 \\
\hline & \begin{tabular}{|l|} 
TT \\
\end{tabular} & 15 & $2.66 \%$ & 8 & $2.57 \%$ & $0.978(0.410-2.337)$ & 0.00 & 0.961 \\
\hline rs28947200 & $\mathrm{C}$ & 1047 & $92.98 \%$ & 575 & $92.44 \%$ & & & \\
\hline & \begin{tabular}{|l|}
$\mathrm{T}$ \\
\end{tabular} & 79 & $7.02 \%$ & 47 & $7.56 \%$ & $1.083(0.744-1.576)$ & 0.170 & 0.676 \\
\hline & TT + CT vs CC & & & & & $1.118(0.731-1.709)$ & 0.260 & 0.606 \\
\hline & TT vs $\mathrm{CT}+\mathrm{CC}$ & & & & & $1.037(0.435-2.473)$ & 0.010 & 0.935 \\
\hline & GG & 522 & $92.72 \%$ & 274 & $88.10 \%$ & Ref & & \\
\hline & AG & 29 & $5.15 \%$ & 25 & $8.04 \%$ & $1.642(0.943-2.860)$ & 3.130 & 0.077 \\
\hline & AA & 12 & $2.13 \%$ & 12 & $3.86 \%$ & $1.905(0.845-4.297)$ & 2.490 & 0.115 \\
\hline rs4392270 & G & 1073 & $95.29 \%$ & 573 & $92.12 \%$ & 1721 (1 $100-07)$ & & \\
\hline & A & 53 & $4.71 \%$ & 49 & $7.88 \%$ & $1.731(1.159-2.587)$ & 7.330 & 0.007 \\
\hline & $\mathrm{AA}+\mathrm{AG}$ vs GG & & & & & $1.719(1.077-2.745)$ & 5.250 & 0.022 \\
\hline & $\mathrm{AA}$ vs $\mathrm{AG}+\mathrm{GG}$ & & & & & $0.543(0.241-1.223)$ & 2.240 & 0.135 \\
\hline
\end{tabular}




\begin{tabular}{|c|c|c|c|c|c|c|c|c|}
\hline SNPs & $\begin{array}{l}\text { Genotype/Allele } \\
\text { distribution }\end{array}$ & $\begin{array}{l}\text { Inactive } \\
(\mathrm{n}=563)\end{array}$ & $\%$ & $\begin{array}{l}\text { Active, cirrhosis } \\
\text { and } \operatorname{HCC}(n=311)\end{array}$ & $\%$ & OR (95\% C.I.) & $x^{2}$ & p-value \\
\hline \multirow{7}{*}{ rs4849133 } & TT & 463 & $82.24 \%$ & 243 & $78.14 \%$ & Ref & & \\
\hline & CT & 65 & $11.55 \%$ & 54 & $17.36 \%$ & $1.583(1.069-2.345)$ & 5.310 & 0.021 \\
\hline & $\mathrm{CC}$ & 35 & $6.22 \%$ & 14 & $4.50 \%$ & $0.762(0.402-1.444)$ & 0.70 & 0.404 \\
\hline & \begin{tabular}{|l|}
$\mathrm{T}$ \\
\end{tabular} & 991 & $88.01 \%$ & 540 & $86.82 \%$ & \multirow{2}{*}{$1.115(0.831-1.495)$} & \multirow{2}{*}{0.530} & \multirow{2}{*}{0.468} \\
\hline & C & 135 & $11.99 \%$ & 82 & $13.18 \%$ & & & \\
\hline & $\mathrm{CC}+\mathrm{CT}$ vs TT & & & & & $1.296(0.918-1.829)$ & 2.170 & 0.141 \\
\hline & $\mathrm{CC}$ vs CT + TT & & & & & $1.406(0.745-2.656)$ & 1.110 & 0.291 \\
\hline \multirow{7}{*}{ rs 2708973} & GG & 512 & $90.94 \%$ & 275 & $88.42 \%$ & Ref & & \\
\hline & AG & 39 & $6.93 \%$ & 26 & $8.36 \%$ & $1.241(0.740-2.082)$ & 0.670 & 0.412 \\
\hline & AA & 12 & $2.13 \%$ & 10 & $3.22 \%$ & $1.552(0.662-3.637)$ & 1.040 & 0.309 \\
\hline & G & 1063 & $94.40 \%$ & 576 & $92.60 \%$ & \multirow{2}{*}{$1.347(0.909-1.997)$} & \multirow{2}{*}{2.220} & \multirow{2}{*}{0.136} \\
\hline & A & 63 & $5.60 \%$ & 46 & $7.40 \%$ & & & \\
\hline & $\mathrm{AA}+\mathrm{AG}$ vs GG & & & & & $1.314(0.837-2.063)$ & 1.520 & 0.234 \\
\hline & $\mathrm{AA}$ vs $\mathrm{AG}+\mathrm{GG}$ & & & & & $0.656(0.280-1.535)$ & 0.960 & 0.327 \\
\hline
\end{tabular}

Table 4. Comparison of genotypic distributions between the inactive group and patients with active HBV, cirrhosis and HCC patients. Bold indicates significance.

\begin{tabular}{|c|c|c|c|c|c|c|c|c|}
\hline SNPs & $\begin{array}{l}\text { Genotype/Allele } \\
\text { distribution }\end{array}$ & $\begin{array}{l}\text { Active } \\
(\mathrm{n}=217)\end{array}$ & $\%$ & $\begin{array}{l}\text { Cirrhosis } \\
(\mathrm{n}=64)\end{array}$ & $\%$ & OR (95\% C.I.) & $x^{2}$ & p-value \\
\hline \multirow{7}{*}{ rs2723176 } & $\mathrm{CC}$ & 195 & $89.86 \%$ & 55 & $85.94 \%$ & Ref & & \\
\hline & $\mathrm{AC}$ & 21 & $9.68 \%$ & 9 & $14.06 \%$ & $1.519(0.658-3.506)$ & 0.970 & 0.324 \\
\hline & $\mathrm{AA}$ & 1 & $0.46 \%$ & 0 & $0.00 \%$ & $1.174(0.047-29.225)$ & 0.280 & 0.596 \\
\hline & $\mathrm{C}$ & 411 & $94.70 \%$ & 119 & $92.97 \%$ & \multirow{2}{*}{$1.351(0.609-2.999)$} & \multirow{2}{*}{0.550} & \multirow{2}{*}{0.457} \\
\hline & A & 23 & $5.30 \%$ & 9 & $7.03 \%$ & & & \\
\hline & $\mathrm{AA}+\mathrm{AC}$ vs $\mathrm{CC}$ & & & & & $1.450(0.632-3.330)$ & 0.780 & 0.378 \\
\hline & $\mathrm{AA}$ vs $\mathrm{AC}+\mathrm{CC}$ & & & & & $0.894(0.036-22.206)$ & 0.30 & 0.586 \\
\hline \multirow{7}{*}{ rs2723175 } & GG & 148 & $68.20 \%$ & 46 & $71.88 \%$ & Ref & & \\
\hline & AG & 61 & $28.11 \%$ & 14 & $21.88 \%$ & $0.738(0.378-1.441)$ & 0.790 & 0.373 \\
\hline & $\mathrm{AA}$ & 8 & $3.69 \%$ & 4 & $6.25 \%$ & $1.609(0.463-5.587)$ & 0.570 & 0.451 \\
\hline & G & 357 & $82.26 \%$ & 106 & $82.81 \%$ & \multirow{2}{*}{$0.962(0.571-1.620)$} & \multirow{2}{*}{0.020} & \multirow{2}{*}{0.885} \\
\hline & A & 77 & $17.74 \%$ & 22 & $17.19 \%$ & & & \\
\hline & $\mathrm{AA}+\mathrm{AG}$ vs $\mathrm{GG}$ & & & & & $0.839(0.454-1.553)$ & 0.310 & 0.576 \\
\hline & AA vs $A G+G G$ & & & & & $0.574(0.167-1.972)$ & 0.790 & 0.373 \\
\hline \multirow{7}{*}{ rs2723186 } & GG & 193 & $88.94 \%$ & 55 & $85.94 \%$ & Ref & & \\
\hline & AG & 15 & $6.91 \%$ & 6 & $9.38 \%$ & $1.404(0.520-3.789)$ & 0.450 & 0.502 \\
\hline & $\mathrm{AA}$ & 9 & $4.15 \%$ & 3 & $4.69 \%$ & $1.170(0.306-4.470)$ & 0.050 & 0.818 \\
\hline & G & 401 & $92.40 \%$ & 116 & $90.63 \%$ & \multirow{2}{*}{$1.257(0.629-2.512)$} & \multirow{2}{*}{0.420} & \multirow{2}{*}{0.516} \\
\hline & A & 33 & $7.60 \%$ & 12 & $9.38 \%$ & & & \\
\hline & $\mathrm{AA}+\mathrm{AG}$ vs $\mathrm{GG}$ & & & & & $1.316(0.578-2.995)$ & 0.430 & 0.512 \\
\hline & $\mathrm{AA}$ vs $\mathrm{AG}+\mathrm{GG}$ & & & & & $0.880(0.231-3.351)$ & 0.040 & 0.851 \\
\hline \multirow{7}{*}{ rs 2723168} & AA & 9 & $4.15 \%$ & 3 & $4.69 \%$ & Ref & & \\
\hline & AG & 207 & $95.39 \%$ & 61 & $95.31 \%$ & $0.884(0.232-3.368)$ & 0.030 & 0.857 \\
\hline & GG & 1 & $0.46 \%$ & 0 & $0.00 \%$ & $0.905(0.029-27.858)$ & 0.330 & 0.569 \\
\hline & $\mathrm{A}$ & 225 & $51.84 \%$ & 67 & $52.34 \%$ & \multirow{2}{*}{$0.980(0.661-1.454)$} & \multirow{2}{*}{0.010} & \multirow{2}{*}{0.921} \\
\hline & G & 209 & $48.16 \%$ & 61 & $47.66 \%$ & & & \\
\hline & GG + AG vs AA & & & & & $0.880(0.231-3.351)$ & 0.040 & 0.851 \\
\hline & GG vs AG+AA & & & & & $0.894(0.036-22.206)$ & 0.30 & 0.586 \\
\hline \multirow{7}{*}{ rs 4364030} & CC & 115 & $53.00 \%$ & 30 & $46.88 \%$ & Ref & & \\
\hline & CG & 81 & $37.33 \%$ & 25 & $39.06 \%$ & $1.183(0.648-2.160)$ & 0.30 & 0.584 \\
\hline & GG & 21 & $9.68 \%$ & 9 & $14.06 \%$ & $1.643(0.683-3.954)$ & 1.240 & 0.265 \\
\hline & $\mathrm{C}$ & 311 & $71.66 \%$ & 85 & $66.41 \%$ & \multirow{2}{*}{$1.279(0.839-1.951)$} & \multirow{2}{*}{1.310} & \multirow{2}{*}{0.252} \\
\hline & G & 123 & $28.34 \%$ & 43 & $33.59 \%$ & & & \\
\hline & GG + CG vs CC & & & & & $1.278(0.731-2.234)$ & 0.740 & 0.389 \\
\hline & GG vs CG + CC & & & & & $0.655(0.284-1.511)$ & 1.00 & 0.318 \\
\hline
\end{tabular}




\begin{tabular}{|c|c|c|c|c|c|c|c|c|}
\hline SNPs & $\begin{array}{l}\text { Genotype/Allele } \\
\text { distribution }\end{array}$ & $\begin{array}{l}\text { Active } \\
(\mathrm{n}=217)\end{array}$ & $\%$ & $\begin{array}{l}\text { Cirrhosis } \\
(\mathrm{n}=64)\end{array}$ & $\%$ & OR (95\% C.I.) & $x^{2}$ & p-value \\
\hline \multirow{7}{*}{ rs3811047 } & GG & 73 & $33.64 \%$ & 19 & $29.69 \%$ & Ref & & \\
\hline & AG & 84 & $38.71 \%$ & 29 & $45.31 \%$ & $1.326(0.687-2.561)$ & 0.710 & 0.399 \\
\hline & AA & 60 & $27.65 \%$ & 16 & $25.00 \%$ & $1.025(0.485-2.164)$ & 0.00 & 0.949 \\
\hline & G & 230 & $53.00 \%$ & 67 & $52.34 \%$ & \multirow{2}{*}{$1.026(0.692-1.523)$} & \multirow{2}{*}{0.020} & \multirow{2}{*}{0.897} \\
\hline & A & 204 & $47.00 \%$ & 61 & $47.66 \%$ & & & \\
\hline & $\mathrm{AA}+\mathrm{AG}$ vs GG & & & & & $1.201(0.655-2.200)$ & 0.350 & 0.554 \\
\hline & $\mathrm{AA}$ vs $\mathrm{AG}+\mathrm{GG}$ & & & & & $1.146(0.605-2.173)$ & 0.180 & 0.675 \\
\hline \multirow{7}{*}{ rs 28947200} & CC & 194 & $89.40 \%$ & 55 & $85.94 \%$ & Ref & & \\
\hline & CT & 17 & $7.83 \%$ & 8 & $12.50 \%$ & $1.660(0.680-4.050)$ & 1.260 & 0.262 \\
\hline & TT & 6 & $2.76 \%$ & 1 & $1.56 \%$ & $0.588(0.069-4.987)$ & 0.240 & 0.622 \\
\hline & C & 405 & $93.32 \%$ & 118 & $92.19 \%$ & \multirow{2}{*}{$1.184(0.560-2.499)$} & \multirow{2}{*}{0.20} & \multirow{2}{*}{0.658} \\
\hline & $\mathrm{T}$ & 29 & $6.68 \%$ & 10 & $7.81 \%$ & & & \\
\hline & $\mathrm{TT}+\mathrm{CT}$ vs CC & & & & & $1.380(0.604-3.155)$ & 0.590 & 0.443 \\
\hline & $\mathrm{TT}$ vs $\mathrm{CT}+\mathrm{CC}$ & & & & & $1.791(0.212-15.160)$ & 0.290 & 0.587 \\
\hline \multirow{7}{*}{ rs4392270 } & GG & 188 & $86.64 \%$ & 58 & $90.63 \%$ & Ref & & \\
\hline & AG & 18 & $8.29 \%$ & 5 & $7.81 \%$ & $0.900(0.320-2.531)$ & 0.040 & 0.842 \\
\hline & $\mathrm{AA}$ & 11 & $5.07 \%$ & 1 & $1.56 \%$ & $0.295(0.037-2.331)$ & 1.510 & 0.219 \\
\hline & G & 394 & $90.78 \%$ & 121 & $94.53 \%$ & \multirow{2}{*}{$0.570(0.249-1.305)$} & \multirow{2}{*}{1.810} & \multirow{2}{*}{0.178} \\
\hline & A & 40 & $9.22 \%$ & 7 & $5.47 \%$ & & & \\
\hline & $\mathrm{AA}+\mathrm{AG}$ vs GG & & & & & $0.671(0.265-1.695)$ & 0.720 & 0.396 \\
\hline & $\mathrm{AA}$ vs $\mathrm{AG}+\mathrm{GG}$ & & & & & $3.364(0.426-26.566)$ & 1.490 & 0.223 \\
\hline \multirow{7}{*}{ rs4849133 } & TT & 177 & $81.57 \%$ & 46 & $71.88 \%$ & Ref & & \\
\hline & CT & 31 & $14.29 \%$ & 16 & $25.00 \%$ & $1.986(1.001-3.940)$ & 3.950 & 0.047 \\
\hline & $\mathrm{CC}$ & 9 & $4.15 \%$ & 2 & $3.13 \%$ & $0.855(0.179-4.094)$ & 0.040 & 0.844 \\
\hline & $\mathrm{T}$ & 385 & $88.71 \%$ & 108 & $84.38 \%$ & \multirow{2}{*}{$1.455(0.829-2.553)$} & \multirow{2}{*}{1.720} & \multirow{2}{*}{0.189} \\
\hline & $\mathrm{C}$ & 49 & $11.29 \%$ & 20 & $15.63 \%$ & & & \\
\hline & $\mathrm{CC}+\mathrm{CT}$ vs TT & & & & & $1.732(0.909-3.297)$ & 2.830 & 0.092 \\
\hline & CC vs CT + TT & & & & & $1.341(0.282-6.372)$ & 0.140 & 0.711 \\
\hline \multirow{7}{*}{ rs2708973 } & GG & 190 & $87.56 \%$ & 59 & $92.19 \%$ & Ref & & \\
\hline & AG & 20 & $9.22 \%$ & 3 & $4.69 \%$ & $0.483(0.139-1.683)$ & 1.360 & 0.244 \\
\hline & $\mathrm{AA}$ & 7 & $3.23 \%$ & 2 & $3.13 \%$ & $0.920(0.186-4.550)$ & 0.010 & 0.919 \\
\hline & G & 400 & $92.17 \%$ & 121 & $94.53 \%$ & \multirow{2}{*}{$0.681(0.294-1.574)$} & \multirow{2}{*}{0.820} & \multirow{2}{*}{0.366} \\
\hline & A & 34 & $7.83 \%$ & 7 & $5.47 \%$ & & & \\
\hline & $\mathrm{AA}+\mathrm{AG}$ vs GG & & & & & $0.596(0.220-1.618)$ & 1.050 & 0.306 \\
\hline & $\mathrm{AA}$ vs $\mathrm{AG}+\mathrm{GG}$ & & & & & $1.033(0.209-5.102)$ & 0.00 & 0.968 \\
\hline
\end{tabular}

Table 5. Comparison of genotypic distributions between the active group and patients with cirrhosis.

Genotype and allele frequency distributions of IL-37 polymorphisms associated with HBV infection and clearance. The genotype distribution and allele frequency for $I L-37$ polymorphisms between the HBV-infected group and control subjects are summarized in Table 2. The major allele homozygous genotype for each SNP was defined as the reference (Ref) genotype. Our results showed that two SNPs within the IL-37 gene (rs2723175 and rs2708973) were significantly associated with a higher risk for HBV infection compared to the healthy controls (Table 2). In particular, both SNPs were associated with the highest risk of HBV infection under the dominant model $(\mathrm{p}<0.0001, \mathrm{OR}=5.895,95 \% \mathrm{CI}=4.216-8.243$ and $\mathrm{p}<0.0001, \mathrm{OR}=2.768,95 \%$ $\mathrm{CI}=1.727-4.438$, respectively). Three other SNPs showed suggestive significance at a nominal p-value threshold (rs4849133, rs28947200, and rs2723186). The TT genotype of rs28947200 was associated with a lower number of patients infected with $\mathrm{HBV}(\mathrm{p}=0.004, \mathrm{OR}=0.462,95 \% \mathrm{CI}=0.268-0.796)$, whereas rs 4849133 was related to the risk of HBV infection under both the dominant and recessive models $(\mathrm{p}=0.015, \mathrm{OR}=1.419,95 \% \mathrm{CI}=1.069$ $1.885 ; \mathrm{p}=0.029, \mathrm{OR}=0.552,95 \% \mathrm{CI}=0.321-0.947$, respectively) compared to healthy controls. The minor allele A of rs2723186 was associated with patients infected with HBV at a nominal p-value, with $\mathrm{OR}=1.424$, $95 \% \mathrm{CI}=1.045-1.939$ and $\mathrm{p}$-value $=0.024$. No significant difference in the genotype and allele distributions of rs2723176, rs2723168, rs4364030, rs3811047, and rs4392270 SNPs was observed in patients infected with HBV compared to the healthy controls (Table 2).

Genotype and allele distribution were also determined in patients infected with HBV and the HBV clearance group due to the natural host immune response. Among the ten $I L-37$ polymorphisms, four SNPs (rs2723176, rs2723186, rs4364030 and rs4392270) were significantly associated with a predisposition for HBV clearance compared to patients with chronic HBV infection (Table 3). The A allele of rs2723176, compared to the C allele, showed the highest correlation with HBV clearance $(\mathrm{p}<0.0001, \mathrm{OR}=0.517,95 \% \mathrm{CI}=0.373-0.716)$. Under the dominant model, there was a significant association for rs2723176 when comparing chronically infected patients 
with the clearance group ( $\mathrm{AA}+\mathrm{AC}$ vs $\mathrm{CC}, \mathrm{p}=0.0002, \mathrm{OR}=0.519,95 \% \mathrm{CI}=0.365-0.738$ ). In addition, the AG genotype of rs2723186, compared to the GG genotype $(\mathrm{p}<0.0001, \mathrm{OR}=0.369,95 \% \mathrm{CI}=0.250-0.543)$ exhibited a decreased risk of HBV infection. An individual carrying the $\mathrm{G}$ minor allele of rs 4364030 showed improved viral clearance, with a p-value of 0.0002 and an OR of 0.716 with a $95 \%$ CI value of $0.601-0.853$. The heterozygous genotype AG of rs 4392270 was positively associated with HBV clearance with a p-value $<0.0001$, and the A allele was found to be associated with a decreased risk of HBV infection at a nominal p-value level $(\mathrm{p}=0.012, \mathrm{OR}=0.667$, $95 \% \mathrm{CI}=0.485-0.918$ ). Similarly, a significant association at a nominal $\mathrm{p}$-value of rs 4849133 heterozygous CT genotype was found, when compared to the dominant TT genotype, with $p=0.017$ and $\mathrm{OR}=0.681$. However, two SNPs, rs2723175 and rs28947200, were associated with an increased risk of HBV infection (Table 3). The rs2723175 AG genotype was found to be associated with patients infected with HBV with a p-value $<0.0001$ $(\mathrm{OR}=10.614$ and $\mathrm{CI}=6.097-18.480)$. The $\mathrm{rs} 28947200 \mathrm{CT}$ genotype was associated with the highest risk of HBV infection $(\mathrm{p}<0.0001, \mathrm{OR}=20.389,95 \% \mathrm{CI}=4.986-83.377)$ when comparing patients with HBV to the clearance group. No significant difference was found between the HBV clearance group and the HBV infected group in the remaining SNPs (Table 3).

Genotype and allele frequency distributions of IL-37 polymorphisms associated with HBV-related liver diseases. Genotypic and allelic distributions were determined in patients characterized as inactive HBsAg carriers and patients infected with HBV who were considered as active carriers including patients who developed cirrhosis and HCC. Only two SNPs were found to be significantly associated with progression to more severe liver abnormalities at a nominal p-value level; rs4392270 with a p-value of 0.007 $(\mathrm{OR}=1.731,95 \% \mathrm{CI}=1.159-2.587)$, and $\mathrm{rs} 4849133$ ( $\mathrm{p}$-value $=0.021, \mathrm{OR}=1.583,95 \% \mathrm{CI}=1.069-2.345)$. No significant difference in the genotype and allele distributions of the other SNPs was observed between the inactive group compared to the group of patients considered as active carriers, cirrhosis and HCC (Table 4).

To assess the influence of $I L-37$ polymorphism on the risk of HBV-mediated liver disease progression to end-stage liver diseases (liver cirrhosis and/or HCC), the genotype and allelic distributions were analyzed between patients actively infected with HBV and the patients diagnosed with liver cirrhosis with and without HCC. The CT genotype of rs 4849133 was found to be nominally significantly associated with progressing to cirrhosis $(\mathrm{p}=0.047, \mathrm{OR}=1.986,95 \% \mathrm{CI}=1.001-3.940)($ Table 5$)$, as well as to cirrhosis with HCC $(\mathrm{p}=0.025$, $\mathrm{OR}=1.990,95 \% \mathrm{CI}=1.082-3.658$ ) (Table 6). No significant difference in the genotype and allele distributions of the other SNPs were observed between patients infected with HBV characterized as active carriers and patients diagnosed with liver cirrhosis or liver cirrhosis with HCC.

Haplotype analysis. The haplotype combinations for the $I L-37$ polymorphisms and their genotypic distribution in $\mathrm{HBV}$-infected patients and the clearance group were determined. The haplotype containing the $\mathrm{C}$ allele of rs4364030, A allele of rs3811047, and C allele of rs2723176 (CAC) (Supplementary Fig. 1) was found to be significantly associated with HBV clearance $(\mathrm{p}<0.0001$, freq. $=0.402)$ (Table 7). Moreover, the distribution of two haplotypes (GGC and CAA) with lower frequencies was found to be significantly different when comparing patients infected with HBV to the clearance group ( $\mathrm{p}<0.0001$, freq. $=0.313 ; \mathrm{p}<0.001$, freq. $=0.055$, respectively) (Table 7).

\section{Discussion}

In the absence of an effective anti-HBV treatment ${ }^{32}$, there is an urgent need for predictive genetic tools to characterize patients with a higher susceptibility to HBV infection, clearance and to HBV-mediated liver diseases. Such genetic screening could support improved therapeutic outcomes ${ }^{32}$. It is well-established that host genetic variations are highly important in the development of HCC in HBV-infected patients. Therefore, it is essential to identify biomarkers for high-risk patients for improved management and treatment. Such markers could be useful in predicting tumor aggressiveness, progression and clinical phenotype. Host genetic markers have been identified for colorectal cancer ${ }^{33}$, breast cancer ${ }^{34}$ and other types of cancer ${ }^{35}$.

As immunity plays a pivotal role in the natural course of HBV, the outcome of the infection, and the pathogenesis of liver disease, the genes encoding inflammatory mediators (e.g., TNF- $\alpha$, TGF- $\beta$, and IL-37) may be prospective candidates to predict the progression of HBV-mediated disease severity ${ }^{36,37}$. Here, we investigated the frequency of genetic variants within the $I L-37$ gene, a recently discovered immune-suppressive cytokine, and determined the degree of association with HBV infection and different levels of HBV-related pathogenesis progression. More specifically, we were interested in identifying any association between the studied SNPs and spontaneous HBV clearance and/or development of severe forms of HBV-associated disease, such as those involving development of liver complications. Among ten $I L-37$ SNPs genotyped, our results revealed that the A allele of rs2723175 was strongly associated with the risk of HBV infection and viral clearance. Additionally, rs4849133 showed a suggestive association with risk for being an active HBsAg carrier and for HBV-mediated end-stage liver disease progression.

Increased risks for HBV infection and HBV-related liver disease are primarily influenced by host factors such as age, gender, BMI, and genetic characteristics ${ }^{38,39}$. In this study, we confirmed the positive influence of these host factors on the increased risk for HBV infection and for HBV-related liver disease. However, on an individual basis, the search for potential predictive genetic risk factors for HBV infection and HBV-mediated end-stage liver disease progression has raised considerable attention in the field of human medical genetics for improved therapeutic management approaches.

In a normal non-infected state, the human liver contains resident antigen-nonspecific immune cells involved in innate immunity, such as natural killer cells, dendritic cells and macrophages named Kupffer cells. The liver also contains cells involved in adaptive immunity including antigen-specific immune T and B cells. Following liver infection with HBV, inflammation occurs due to the production and release of inflammatory cytokines by 


\begin{tabular}{|c|c|c|c|c|c|c|c|c|}
\hline SNPs & $\begin{array}{l}\text { Genotype/Allele } \\
\text { distribution }\end{array}$ & $\begin{array}{l}\text { Active } \\
(\mathrm{n}=217)\end{array}$ & $\%$ & $\begin{array}{l}\text { Cirrhosis + HCC } \\
(\mathrm{n}=94)\end{array}$ & $\%$ & OR (95\% C.I.) & $x^{2}$ & p-value \\
\hline \multirow{7}{*}{ rs2723176 } & CC & 195 & $89.86 \%$ & 81 & $86.17 \%$ & Ref & & \\
\hline & AC & 21 & $9.68 \%$ & 12 & $12.77 \%$ & $1.376(0.647-2.927)$ & 0.690 & 0.406 \\
\hline & AA & 1 & $0.46 \%$ & 1 & $1.06 \%$ & $2.407(0.149-38.956)$ & 0.410 & 0.523 \\
\hline & $\mathrm{C}$ & 411 & $94.70 \%$ & 174 & $92.55 \%$ & \multirow{2}{*}{$1.438(0.723-2.860)$} & \multirow{2}{*}{1.080} & \multirow{2}{*}{0.298} \\
\hline & A & 23 & $5.30 \%$ & 14 & $7.45 \%$ & & & \\
\hline & $\mathrm{AA}+\mathrm{AC}$ vs CC & & & & & $1.423(0.684-2.961)$ & 0.890 & 0.344 \\
\hline & $\mathrm{AA}$ vs $\mathrm{AC}+\mathrm{CC}$ & & & & & $0.431(0.027-6.957)$ & 0.370 & 0.541 \\
\hline \multirow{7}{*}{ rs2723175 } & GG & 148 & $68.20 \%$ & 69 & $73.40 \%$ & Ref & & \\
\hline & AG & 61 & $28.11 \%$ & 21 & $22.34 \%$ & $0.738(0.417-1.309)$ & 1.080 & 0.298 \\
\hline & AA & 8 & $3.69 \%$ & 4 & $4.26 \%$ & $1.072(0.312-3.683)$ & 0.010 & 0.911 \\
\hline & G & 357 & $82.26 \%$ & 159 & $84.57 \%$ & \multirow{2}{*}{$0.846(0.531-1.348)$} & \multirow{2}{*}{0.50} & \multirow{2}{*}{0.480} \\
\hline & A & 77 & $17.74 \%$ & 29 & $15.43 \%$ & & & \\
\hline & $\mathrm{AA}+\mathrm{AG}$ vs GG & & & & & $0.777(0.453-1.333)$ & 0.840 & 0.359 \\
\hline & $\mathrm{AA}$ vs $\mathrm{AG}+\mathrm{GG}$ & & & & & $0.861(0.253-2.933)$ & 0.060 & 0.811 \\
\hline \multirow{7}{*}{ rs2723186 } & GG & 193 & $88.94 \%$ & 80 & $85.11 \%$ & Ref & & \\
\hline & AG & 15 & $6.91 \%$ & 8 & $8.51 \%$ & $1.448(0.609-3.443)$ & 0.710 & 0.401 \\
\hline & AA & 9 & $4.15 \%$ & 5 & $5.32 \%$ & $1.340(0.436-4.124)$ & 0.260 & 0.608 \\
\hline & G & 401 & $92.40 \%$ & 168 & $89.36 \%$ & \multirow[b]{2}{*}{$1.366(0.756-2.470)$} & \multirow{2}{*}{1.070} & \multirow{2}{*}{0.300} \\
\hline & A & 33 & $7.60 \%$ & 18 & $9.57 \%$ & & & \\
\hline & $\mathrm{AA}+\mathrm{AG}$ vs GG & & & & & $1.407(0.693-2.859)$ & 0.90 & 0.343 \\
\hline & $\mathrm{AA}$ vs $\mathrm{AG}+\mathrm{GG}$ & & & & & $0.770(0.251-2.363)$ & 0.210 & 0.647 \\
\hline \multirow{7}{*}{ rs2723168 } & AA & 9 & $4.15 \%$ & 3 & $3.19 \%$ & Ref & & \\
\hline & AG & 207 & $95.39 \%$ & 90 & $95.74 \%$ & $1.304(0.345-4.931)$ & 0.150 & 0.695 \\
\hline & GG & 1 & $0.46 \%$ & 1 & $1.06 \%$ & $3.00(0.140-64.262)$ & 0.530 & 0.468 \\
\hline & $\mathrm{A}$ & 225 & $51.84 \%$ & 96 & $51.06 \%$ & \multirow{2}{*}{$1.032(0.733-1.453)$} & \multirow{2}{*}{0.030} & \multirow{2}{*}{0.858} \\
\hline & G & 209 & $48.16 \%$ & 92 & $48.94 \%$ & & & \\
\hline & GG + AG vs AA & & & & & $1.312(0.347-4.961)$ & 0.160 & 0.688 \\
\hline & GG vs AG + AA & & & & & $0.431(0.027-6.957)$ & 0.370 & 0.541 \\
\hline & CC & 115 & $53.00 \%$ & 45 & $47.87 \%$ & Ref & & \\
\hline & CG & 81 & $37.33 \%$ & 35 & $37.23 \%$ & $1.104(0.653-1.867)$ & 0.14 & 0.711 \\
\hline & GG & 21 & $9.68 \%$ & 14 & $14.89 \%$ & $1.704(0.798-3.639)$ & 1.92 & 0.166 \\
\hline rs4364030 & C & 311 & $71.66 \%$ & 125 & $66.49 \%$ & $1274(0.882-1841)$ & 167 & 0196 \\
\hline & G & 123 & $28.34 \%$ & 63 & $33.51 \%$ & $1.274(0.882-1.841)$ & 1.67 & 0.196 \\
\hline & GG + CG vs CC & & & & & $1.228(0.756-1.993)$ & 0.690 & 0.406 \\
\hline & GG vs CG + CC & & & & & $0.612(0.297-1.264)$ & 1.790 & 0.181 \\
\hline & GG & 73 & $33.64 \%$ & 31 & $32.98 \%$ & Ref & & \\
\hline & AG & 84 & $38.71 \%$ & 38 & $40.43 \%$ & $1.065(0.603-1.881)$ & 0.050 & 0.827 \\
\hline & AA & 60 & $27.65 \%$ & 25 & $26.60 \%$ & $0.981(0.524-1.838)$ & 0.00 & 0.953 \\
\hline rs3811047 & G & 230 & $53.00 \%$ & 100 & $53.19 \%$ & $002(0704,1309$ & Oano & 0.66 \\
\hline & A & 204 & $47.00 \%$ & 88 & $46.81 \%$ & $0.992(0.704-1.398)$ & 0.00 & 0.964 \\
\hline & $\mathrm{AA}+\mathrm{AG}$ vs GG & & & & & $1.030(0.616-1.723)$ & 0.010 & 0.909 \\
\hline & AA vs $A G+G G$ & & & & & $1.055(0.611-1.820)$ & 0.040 & 0.848 \\
\hline & $\mathrm{CC}$ & 194 & $89.40 \%$ & 78 & $82.98 \%$ & Ref & & \\
\hline & CT & 17 & $7.83 \%$ & 14 & $14.89 \%$ & $2.048(0.963-4.356)$ & 3.580 & 0.059 \\
\hline & TT & 6 & $2.76 \%$ & 2 & $2.13 \%$ & $0.829(0.164-4.197)$ & 0.050 & 0.820 \\
\hline rs28947200 & $\mathrm{C}$ & 405 & $93.32 \%$ & 170 & $90.43 \%$ & & & \\
\hline & $\mathrm{T}$ & 29 & $6.68 \%$ & 18 & $9.57 \%$ & $1.479(0.800-2.735)$ & 1.570 & 0.210 \\
\hline & TT + CT vs CC & & & & & $1.730(0.868-3.450)$ & 2.470 & 0.116 \\
\hline & TT vs CT + CC & & & & & $1.308(0.259-6.603)$ & 0.110 & 0.744 \\
\hline & GG & 188 & $86.64 \%$ & 86 & $91.49 \%$ & Ref & & \\
\hline & AG & 18 & $8.29 \%$ & 7 & $7.45 \%$ & $0.850(0.342-2.11)$ & 0.120 & 0.726 \\
\hline & AA & 11 & $5.07 \%$ & 1 & $1.06 \%$ & $0.199(0.025-1.564)$ & 2.890 & 0.089 \\
\hline rs 4392270 & G & 394 & $90.78 \%$ & 179 & $95.21 \%$ & & & \\
\hline & A & 40 & $9.22 \%$ & 9 & $4.79 \%$ & $0.495(0.235-1.043)$ & 3.550 & 0.059 \\
\hline & $\mathrm{AA}+\mathrm{AG}$ vs GG & & & & & $0.603(0.265-1.374)$ & 1.470 & 0.225 \\
\hline & $\mathrm{AA}$ vs $\mathrm{AG}+\mathrm{GG}$ & & & & & $4.966(0.632-39.030)$ & 2.840 & 0.092 \\
\hline
\end{tabular}




\begin{tabular}{|c|c|c|c|c|c|c|c|c|}
\hline SNPs & $\begin{array}{l}\text { Genotype/Allele } \\
\text { distribution }\end{array}$ & $\begin{array}{l}\text { Active } \\
(n=217)\end{array}$ & $\%$ & $\begin{array}{l}\text { Cirrhosis + HCC } \\
(\mathrm{n}=94)\end{array}$ & $\%$ & OR (95\% C.I.) & $x^{2}$ & p-value \\
\hline \multirow{7}{*}{ rs4849133 } & TT & 177 & $81.57 \%$ & 66 & $70.21 \%$ & Ref & & \\
\hline & CT & 31 & $14.29 \%$ & 23 & $24.47 \%$ & $1.990(1.082-3.658)$ & 5.010 & 0.025 \\
\hline & $\mathrm{CC}$ & 9 & $4.15 \%$ & 5 & $5.32 \%$ & $1.490(0.482-4.608)$ & 0.480 & 0.486 \\
\hline & $\mathrm{T}$ & 385 & $88.71 \%$ & 155 & $82.45 \%$ & \multirow{2}{*}{$1.673(1.036-2.701)$} & \multirow{2}{*}{4.50} & \multirow{2}{*}{0.033} \\
\hline & C & 49 & $11.29 \%$ & 33 & $17.55 \%$ & & & \\
\hline & $\mathrm{CC}+\mathrm{CT}$ vs TT & & & & & $1.877(1.073-3.285)$ & 4.950 & 0.026 \\
\hline & CC vs CT + TT & & & & & $0.770(0.251-2.363)$ & 0.210 & 0.647 \\
\hline \multirow{7}{*}{ rs2708973 } & GG & 190 & $87.56 \%$ & 85 & $90.43 \%$ & Ref & & \\
\hline & AG & 20 & $9.22 \%$ & 6 & $6.38 \%$ & $0.671(0.260-1.730)$ & 0.690 & 0.406 \\
\hline & $\mathrm{AA}$ & 7 & $3.23 \%$ & 3 & $3.19 \%$ & $0.958(0.242-3.794)$ & 0.00 & 0.951 \\
\hline & G & 400 & $92.17 \%$ & 176 & $93.62 \%$ & \multirow{2}{*}{$0.802(0.406-1.586)$} & \multirow{2}{*}{0.40} & \multirow{2}{*}{0.525} \\
\hline & A & 34 & $7.83 \%$ & 12 & $6.38 \%$ & & & \\
\hline & $\mathrm{AA}+\mathrm{AG}$ vs GG & & & & & $0.745(0.336-1.653)$ & 0.530 & 0.468 \\
\hline & $\mathrm{AA}$ vs $\mathrm{AG}+\mathrm{GG}$ & & & & & $1.011(0.256-3.998)$ & 0.00 & 0.987 \\
\hline
\end{tabular}

Table 6. Comparison of genotypic distributions between the active group and patients with cirrhosis and HCC.

\begin{tabular}{|l|l|l|l|l|l|l|c|}
\hline \multicolumn{2}{|l|}{ Haplotypes } & Freq. & $\begin{array}{l}\text { Case, Control Ratio } \\
\text { Counts* }\end{array}$ & $\begin{array}{l}\text { Case, Control } \\
\text { Frequencies }\end{array}$ & $\begin{array}{l}\text { Chi } \\
\text { Square }\end{array}$ & p-value \\
\hline rs4364030 & rs3811047 & rs2723176 & & & & & \\
\hline C & A & C & 0.402 & $748.1: 999.9,275.4: 524.6$ & $0.428,0.344$ & 16.008 & $<\mathbf{0 . 0 0 0 1}$ \\
\hline G & G & C & 0.313 & $503.5: 1244.5,293.4: 506.6$ & $0.288,0.367$ & 15.81 & $<\mathbf{0 . 0 0 0 1}$ \\
\hline C & G & C & 0.21 & $383.4: 1364.6,151.9: 648.1$ & $0.219,0.190$ & 2.883 & 0.089 \\
\hline C & A & A & 0.055 & $77.6: 1670.4,62.1: 737.9$ & $0.044,0.078$ & 11.724 & $<\mathbf{0 . 0 0 1}$ \\
\hline G & A & C & 0.014 & $28.0: 1720.0,7.4: 792.6$ & $0.016,0.009$ & 1.858 & 0.173 \\
\hline
\end{tabular}

Table 7. Haplotype frequencies of $I L-37$ between the clearance group and patients infected with HBV. ${ }^{*}$ Case $=$ HBV-Infected patients, Control $=$ Clearance group. Bold indicate significance.

hepatocytes and immune cells following the binding of the C-terminal domain of HBV core proteins to membrane heparan sulfate on the cell surface ${ }^{19,40}$. It has been reported that IL-37 inhibits various functions, such as antigen presentation ${ }^{21}$, macrophage activation ${ }^{41}$, and cytokine production ${ }^{42}$. Here, among the ten $I L-37$ SNPs screened, half show a significant association of susceptibility to HBV infection, including rs2723175, which in our study was found to be strongly disease-associated variants through the heterozygous AG genotype with a dominance of risk allele "A". Among the ten $I L-37$ SNPs analyzed in the Saudi population, three SNPs, rs2723176, rs2723186, rs3811047, have been reported to have a genetic predisposition for auto-immune thyroid disease in the Chinese population ${ }^{28}$. Polymorphisms of other anti-inflammatory cytokines such as IL-10 and IL-4 are also reported to be strongly associated with the outcome of $\mathrm{HBV}$ infection ${ }^{43,44}$.

HBV clearance and clinical recovery occur mainly through the induction of effective intrahepatic virus-specific $\mathrm{CD}^{+}$and $\mathrm{CD}^{+}{ }^{+} \mathrm{T}$ cells ${ }^{45}$. Thus, the loss of $\mathrm{T}$-cell activity or a decrease in T-cell ability to produce key antiviral and immune stimulatory cytokines shifts from HBV viral clearance toward HBV viral persistence. In comparison with patients undergoing HBV clearance, two IL-37 polymorphisms (rs2723175 and rs28947200) were strongly associated with HBV infection, suggesting an inhibition of T-cell activity which reinforces the immunosuppressive effects of IL-37. IL-37 has also been demonstrated to inhibit antigen-specific T-cell proliferation ${ }^{46}$. In addition, IL-37 has been demonstrated to be expressed by immunosuppressive regulatory $\mathrm{T}$ cells $\mathrm{s}^{47}$, which are correlated with chronic HBV infection and demonstrated to exert an active influence on HBV clearance ${ }^{48}$. However, six out of ten polymorphisms, including rs4849133, were associated with HBV clearance compared to HBV infection. This strong positive correlation of IL-37 polymorphisms with HBV clearance suggests an inability of $I L-37$ genetic variants to inactivate T-cell activity and subsequently offering a protective role of $I L-37$ polymorphisms for HBV clearance. In a normal non-infected person, low levels of steady state IL-37 mRNA and protein are found expressed in monocytes, dendritic cells, and plasma cells. However, under inflammatory conditions, $I L-37$ gene expression is stimulated with pro-inflammatory cytokines, such as IL- $1 \beta$, IL-18, TNF- $\alpha$, IFN- $\gamma$, and TGF- $\beta$, or Toll-like receptor (TLR) ligands, and downregulated by IL-12, IL-32, and GM-CSF plus IL-4. This immune mechanism suppresses the proinflammatory cytokines IL-1 $\beta$, IL- $1 \alpha$, IL-6, M-CSF, and GM-CSF but not the anti-inflammatory cytokines IL-10 and IL-1 Ra ${ }^{22}$. Thus, a comparative study of $I L-37$ genetic variants in patients infected with HBV for IL-37 production, at both the transcript and protein levels, will clarify the role of IL-37 in HBV clearance and persistence.

Globally, inactive HBsAg carriers form the largest group in chronic HBV-infected patients, indicating the tolerogenic status of HBV immunopathogenesis as patients infected with HBV do not display any discernable clinical disease. In contrast to inactive HBsAg carriers, the active carriers contain a high level of serum HBV 
DNA and circulating serum HBeAg with a high risk for developing liver cirrhosis and HCC. In this study, among the ten genetic variants for the IL-37 gene, only one SNP, rs4849133 with the CT genotype, showed a suggestive association with the risk for active HBsAg carriers compared to patients with inactive HBV infection. These findings are not surprising as the difference between inactive and active carriers based on the viral production of serum HBV DNA copies without involving the host's innate immune system. However, we previously reported that the haplotype of the CXCR1, a receptor for T-cell chemo-attractant cytokines such as IL-8, named Haplo-2 (AC genotype), was significantly associated with HBsAg carrier status ${ }^{49}$, confirming the important influence of the adaptive T-cell immune system on the HBsAg carrier status for differences in IL-37. Although a previous study has reported that an increased serum IL-37 in patients with chronic HBV infection was positively correlated with liver damage ${ }^{24}$, we only identified the SNP rs4849133 CT genotype as being associated with the susceptibility to end-stage liver disease progression in patients infected with HBV when compared to active carriers infected with HBV. Recently, IL-37 has been described to exhibit anti-tumor activity through chemo-attraction of $\mathrm{CD}^{2} 7^{+}$natural killer (NK) cells, inhibiting HCC development ${ }^{23}$. Thus, patients infected with HBV harboring $I L-37$ SNP rs4849133 might fail in the production of active IL-37 protein, which may explain the increased risk for HCC progression. Furthermore, no mutation within $I L-37$ gene has been found in all the HCC cases described in The Cancer Genome Atlas - Liver Hepatocellular Carcinoma project (TCGA-LIHC). However, a modulation of the HCC tumor immune milieu including the depletion of neutrophils and activated macrophages, main sources of IL-37, have been recently reported in a TCGA-LIHC subset of HBV/HCV-infected patients only ${ }^{50}$; which could also explain the decrease of IL-37 production contributing to HCC development and progression.

This study is limited by the fact that the sample sizes in the cirrhosis and HCC groups were small. Also, this study does not include in the final analysis some important factors, such as treatment protocol, treatment outcome and duration of the infection, well known to impact the course of HBV infection. Similarly, survival analyses were not conducted in our study due the cross-sectional feature of this study. Additional studies are required to include such analyses and validate these results. In conclusion, these findings suggest that $I L-37$ polymorphisms may not only be implicated in the development of HCC but may also be involved in HBV infection and in determining different clinical outcomes of HBV infection, including active chronic HBV infection and low viremic "inactive" HBsAg carrier status.

\section{References}

1. Oh, I. S. \& Park, S. H. Immune-mediated Liver Injury in Hepatitis B Virus Infection. Immune Netw 15, 191-198, https://doi. org/10.4110/in.2015.15.4.191 (2015).

2. Franco, E. et al. Hepatitis B: Epidemiology and prevention in developing countries. World J Hepatol 4, 74-80, https://doi. org/10.4254/wjh.v4.i3.74 (2012).

3. Khanna, R., Gupta, E. \& Alam, S. Reverse vertical transmission of hepatitis-B from transfusion-infected children to biological mothers. Indian Pediatr 51, 739-741 (2014).

4. Abdo, A. A., Sanai, F. M. \& Al-Faleh, F. Z. Epidemiology of viral hepatitis in Saudi Arabia: are we off the hook? Saudi J Gastroenterol 18, 349-357, https://doi.org/10.4103/1319-3767.103425 (2012).

5. Schuch, A., Hoh, A. \& Thimme, R. The role of natural killer cells and CD8(+) T cells in hepatitis B virus infection. Front Immunol 5, 258, https://doi.org/10.3389/fimmu.2014.00258 (2014).

6. Thimme, R. et al. $\mathrm{CD} 8(+) \mathrm{T}$ cells mediate viral clearance and disease pathogenesis during acute hepatitis B virus infection. $J$ Virol 77, 68-76 (2003).

7. Beasley, R. P. et al. Prevention of perinatally transmitted hepatitis B virus infections with hepatitis B immune globulin and hepatitis B vaccine. Lancet 2, 1099-1102 (1983).

8. Jonas, M. M. et al. Treatment of children with chronic hepatitis B virus infection in the United States: patient selection and therapeutic options. Hepatology 52, 2192-2205, https://doi.org/10.1002/hep.23934 (2010).

9. Mason, W. S. et al. The amount of hepatocyte turnover that occurred during resolution of transient hepadnavirus infections was lower when virus replication was inhibited with entecavir. J Virol 83, 1778-1789, https://doi.org/10.1128/JVI.01587-08 (2009).

10. Seeger, C. \& Mason, W. S. Molecular biology of hepatitis B virus infection. Virology 479-480, 672-686, https://doi.org/10.1016/j. virol.2015.02.031 (2015).

11. Bertoletti, A. \& Hong, M. Age-Dependent Immune Events during HBV Infection from Birth to Adulthood: An Alternative Interpretation. Front Immunol 5, 441, https://doi.org/10.3389/fimmu.2014.00441 (2014).

12. Chan, H. L. et al. Viral genotype and hepatitis B virus DNA levels are correlated with histological liver damage in HBeAg-negative chronic hepatitis B virus infection. Am J Gastroenterol 97, 406-412, https://doi.org/10.1111/j.1572-0241.2002.05478.x (2002).

13. Francisci, D., Baldelli, F., Papili, R., Stagni, G. \& Pauluzzi, S. Prevalence of HBV, HDV and HCV hepatitis markers in HIV-positive patients. Eur J Epidemiol 11, 123-126 (1995).

14. Marcellin, P. et al. Serum Levels of Hepatitis B Surface Antigen Predict Severity of Fibrosis in Patients With E Antigen-Positive Chronic Hepatitis B. Clin Gastroenterol Hepatol 13, 1532-1539 e1531, https://doi.org/10.1016/j.cgh.2014.12.017 (2015).

15. Stroffolini, T. et al. Gender differences in chronic HBsAg carriers in Italy: Evidence for the independent role of male sex in severity of liver disease. J Med Virol 87, 1899-1903, https://doi.org/10.1002/jmv.24243 (2015).

16. Zeng, Z. et al. A population-based study to investigate host genetic factors associated with hepatitis B infection and pathogenesis in the Chinese population. BMC Infect Dis 8, 1, https://doi.org/10.1186/1471-2334-8-1 (2008).

17. Trehanpati, N. \& Vyas, A. K. Immune Regulation by T Regulatory Cells in Hepatitis B Virus-Related Inflammation and Cancer. Scand J Immunol 85, 175-181, https://doi.org/10.1111/sji.12524 (2017).

18. Wang, M., Xi, D. \& Ning, Q. Virus-induced hepatocellular carcinoma with special emphasis on HBV. Hepatol Int 11, 171-180, https://doi.org/10.1007/s12072-016-9779-5 (2017).

19. T, K. et al. HBV core protein enhances cytokine production. Diseases 3, 213-220 (2015).

20. Schulze, A., Gripon, P. \& Urban, S. Hepatitis B virus infection initiates with a large surface protein-dependent binding to heparan sulfate proteoglycans. Hepatology 46, 1759-1768, https://doi.org/10.1002/hep.21896 (2007).

21. Luo, Y. et al. Suppression of antigen-specific adaptive immunity by IL-37 via induction of tolerogenic dendritic cells. Proc Natl Acad Sci USA 111, 15178-15183, https://doi.org/10.1073/pnas.1416714111 (2014).

22. Nold, M. F. et al. IL-37 is a fundamental inhibitor of innate immunity. Nat Immunol 11, 1014-1022, https://doi.org/10.1038/ni.1944 (2010).

23. Zhao, J. J. et al. Interleukin-37 mediates the antitumor activity in hepatocellular carcinoma: role for CD57+ NK cells. Sci Rep 4, 5177, https://doi.org/10.1038/srep05177 (2014). 
24. Li, C. et al. Serum interleukin-37 concentrations and HBeAg seroconversion in chronic HBV patients during telbivudine treatment. J Interferon Cytokine Res 33, 612-618, https://doi.org/10.1089/jir.2013.0001 (2013).

25. Allam, G. et al. Association of IL-37 gene polymorphisms with susceptibility to tuberculosis in Saudi subjects. Microbiol Immunol 60, 778-786, https://doi.org/10.1111/1348-0421.12444 (2016).

26. Liu, H. et al. IL-37 Confers Protection against Mycobacterial Infection Involving Suppressing Inflammation and Modulating T Cell Activation. PLoS One 12, e0169922, https://doi.org/10.1371/journal.pone.0169922 (2017).

27. Yin, D. et al. Genomic Variant in IL-37 Confers A Significant Risk of Coronary Artery Disease. Sci Rep 7, 42175, https://doi. org/10.1038/srep42175 (2017).

28. Yan, N. et al. Polymorphism of IL37 gene as a protective factor for autoimmune thyroid disease. J Mol Endocrinol 55, 209-218, https://doi.org/10.1530/JME-15-0144 (2015).

29. MOH. A review of health situation. The Annual Health Statistics Book. Ministry of Health, Saudi Arabia., 107-123 (2016).

30. Carlson, C. S. et al. Selecting a maximally informative set of single-nucleotide polymorphisms for association analyses using linkage disequilibrium. Am J Hum Genet 74, 106-120, https://doi.org/10.1086/381000 (2004).

31. Xu, Z., Kaplan, N. L. \& Taylor, J. A. TAGster: efficient selection of LD tag SNPs in single or multiple populations. Bioinformatics 23, 3254-3255, https://doi.org/10.1093/bioinformatics/btm426 (2007).

32. J, C. et al. New insights into hepatitis B virus biology and implications for novel antiviral strategies. Natl Sci Rev 2, 296-313 (2015).

33. Gao, S. et al. Identification and Construction of Combinatory Cancer Hallmark-Based Gene Signature Sets to Predict Recurrence and Chemotherapy Benefit in Stage II Colorectal Cancer. JAMA oncology 2, 37-45, https://doi.org/10.1001/jamaoncol.2015.3413 (2016).

34. McGee, S. R., Tibiche, C., Trifiro, M. \& Wang, E. Network Analysis Reveals A Signaling Regulatory Loop in the PIK3CA-mutated Breast Cancer Predicting Survival Outcome. Genomics, proteomics \& bioinformatics 15, 121-129, https://doi.org/10.1016/j. gpb.2017.02.002 (2017).

35. Wang, E. et al. Predictive genomics: a cancer hallmark network framework for predicting tumor clinical phenotypes using genome sequencing data. Seminars in cancer biology 30, 4-12, https://doi.org/10.1016/j.semcancer.2014.04.002 (2015).

36. Li, X., Liu, X., Tian, L. \& Chen, Y. Cytokine-Mediated Immunopathogenesis of Hepatitis B Virus Infections. Clin Rev Allergy Immunol 50, 41-54, https://doi.org/10.1007/s12016-014-8465-4 (2016).

37. Yang, Q., Shi, Y., Yang, Y., Lou, G. \& Chen, Z. The sterile inflammation in the exacerbation of HBV-associated liver injury. Mediators Inflamm 2015, 508681, https://doi.org/10.1155/2015/508681 (2015).

38. Matsuura, K., Isogawa, M. \& Tanaka, Y. Host genetic variants influencing the clinical course of hepatitis B virus infection. J Med Virol 88, 371-379, https://doi.org/10.1002/jmv.24350 (2016).

39. McMahon, B. J. The natural history of chronic hepatitis B virus infection. Hepatology 49, S45-55, https://doi.org/10.1002/hep.22898 (2009).

40. YJ, L. et al. Hepatitis B virus core antigen determines viral persistence in a C57BL/6 mouse model. Proc Natl Acad Sci. USA 107, 9340-9345 (2014).

41. Li, S. et al. Extracellular forms of IL-37 inhibit innate inflammation in vitro and in vivo but require the IL-1 family decoy receptor IL-1R8. Proc Natl Acad Sci USA 112, 2497-2502, https://doi.org/10.1073/pnas.1424626112 (2015).

42. Ye, L. et al. IL-37 inhibits the production of inflammatory cytokines in peripheral blood mononuclear cells of patients with systemic lupus erythematosus: its correlation with disease activity. J Transl Med 12, 69, https://doi.org/10.1186/1479-5876-12-69 (2014).

43. Z, W. et al. Association between IL-4 polymorphisms and risk of liver disease: an updated meta-analysis. Medicine (Baltimore) 94, e1435 (2015)

44. Gao, L. et al. Association of IL-10 polymorphisms with hepatitis B virus infection and outcome in Han population. Eur J Med Res 21, 23, https://doi.org/10.1186/s40001-016-0218-9 (2016).

45. Tzeng, H. T. et al. Tumor necrosis factor-alpha induced by hepatitis B virus core mediating the immune response for hepatitis B viral clearance in mice model. PLoS One 9, e103008, https://doi.org/10.1371/journal.pone.0103008 (2014).

46. Dinarello, C. A. et al. Suppression of innate inflammation and immunity by interleukin-37. Eur J Immunol 46, 1067-1081, https:// doi.org/10.1002/eji.201545828 (2016).

47. Shuai, X. et al. Expression of IL-37 contributes to the immunosuppressive property of human CD4+CD25+ regulatory T cells. Sci Rep 5, 14478, https://doi.org/10.1038/srep14478 (2015).

48. Gao, Y. et al. Circulating FoxP3+ Regulatory T and Interleukin17-Producing Th17 Cells Actively Influence HBV Clearance in De Novo Hepatitis B Virus Infected Patients after Orthotopic Liver Transplantation. PLoS One 10, e0137881, https://doi.org/10.1371/ journal.pone.0137881 (2015).

49. Almajhdi, F. N. et al. Single nucleotide polymorphisms in CXCR1 gene and its association with hepatitis B infected patients in Saudi Arabia. Ann Hepatol 12, 220-227 (2013).

50. Cancer Genome Atlas Research Network, Comprehensive and integrative genomic characterization of hepatocellular carcinoma. Cell 169, 1327-1341 (2017).

\section{Acknowledgements}

This work was supported by King Abdulaziz City for Science and Technology (KACST), National Plan for Science, Biotechnology, and Innovation (NSTIP) (Project number 11-MED1430-20). This study was approved by Research Advisory Council (RAC) of King Faisal Specialist Hospital and Research Centre (KFSHRC), project number 2150008. The support of the Research Center administration at KFSHRC is highly appreciated.

\section{Author Contributions}

Conceived and designed the experiments: A.A. Al-Q., H.I. Al-A., M.N.Al-A., A.A.A., F.M.S. Performed the experiments: M.R.Al-A., D.D.C., M.F.B. Analyzed the data: M.R.Al-A., S.K.Al-A., S.M.N., J.Al-G., A.A.A., A.Al-B., M.B.S., W.K. Al-H., K.A.Al-S., M.Q.K. Contributed reagents/materials/analysis tools: A.A.A., F.M.S., H.I.Al-A., M.Q.K., J.Al-G., W.K.Al-H., K.A.Al-S., M.Q.K. Wrote the paper: A.A.Al-Q., S.M.N., M.N.Al-A.

\section{Additional Information}

Supplementary information accompanies this paper at https://doi.org/10.1038/s41598-019-42808-4.

Competing Interests: The authors declare no competing interests.

Publisher's note: Springer Nature remains neutral with regard to jurisdictional claims in published maps and institutional affiliations. 
(i) Open Access This article is licensed under a Creative Commons Attribution 4.0 International License, which permits use, sharing, adaptation, distribution and reproduction in any medium or format, as long as you give appropriate credit to the original author(s) and the source, provide a link to the Creative Commons license, and indicate if changes were made. The images or other third party material in this article are included in the article's Creative Commons license, unless indicated otherwise in a credit line to the material. If material is not included in the article's Creative Commons license and your intended use is not permitted by statutory regulation or exceeds the permitted use, you will need to obtain permission directly from the copyright holder. To view a copy of this license, visit http://creativecommons.org/licenses/by/4.0/.

(C) The Author(s) 2019 